\title{
On the rational homotopy type of a moduli space of vector bundles over a curve
}

\author{
INDRANIL BisWAS AND VicEnTE MUÑOZ
}

\begin{abstract}
We study the rational homotopy of the moduli space $\mathcal{N}_{X}$ that parametrizes the isomorphism classes of all stable vector bundles of rank two and fixed determinant of odd degree over a compact connected Riemann surface $X$ of genus $g$, with $g \geq 2$. The symplectic group $\operatorname{Aut}\left(H_{1}(X, \mathbb{Z})\right) \cong \operatorname{Sp}(2 g, \mathbb{Z})$ has a natural action on the rational homotopy groups $\pi_{n}\left(\mathcal{N}_{X}\right) \otimes_{\mathbb{Z}} \mathbb{Q}$. We prove that this action extends to an action of $\operatorname{Sp}(2 g, \mathbb{C})$ on $\pi_{n}\left(\mathcal{N}_{X}\right) \otimes_{\mathbb{Z}} \mathbb{C}$. We also show that $\pi_{n}\left(\mathcal{N}_{X}\right) \otimes_{\mathbb{Z}} \mathbb{C}$ is a non-trivial representation of $\operatorname{Sp}(2 g, \mathbb{C}) \cong$ Aut $\left(H_{1}(X, \mathbb{C})\right)$ for all $n \geq 2 g-1$. In particular, $\mathcal{N}_{X}$ is a rationally hyperbolic space. In the special case where $g=2$, for each $n \in \mathbb{N}$, we compute the leading $\operatorname{Sp}(2 g, \mathbb{C})$ representation occurring in $\pi_{n}\left(\mathcal{N}_{X}\right) \otimes_{\mathbb{Z}} \mathbb{C}$.
\end{abstract}

\section{Introduction}

Moduli spaces of vector bundles over curves have been studied from various points of view. The aim here is to initiate investigations of their rational homotopy groups.

Let $X$ be an irreducible smooth projective curve, defined over $\mathbb{C}$, of genus $g$, with $g \geq 2$. Fix a holomorphic line bundle $L_{0}$ over $X$ of degree 1 , and consider the moduli space $\mathcal{N}_{X}$ of stable vector bundles $E \rightarrow X$ of rank two with $\bigwedge^{2} E \cong L_{0}$. This moduli space $\mathcal{N}_{X}$ is an irreducible smooth complex projective variety of complex dimension $3 g-3$ (see [19]).

The mapping class group of $X$ acts in a natural way on the cohomology algebra $H^{*}\left(\mathcal{N}_{X}, \mathbb{Q}\right)$ of $\mathcal{N}_{X}$. This action actually factors through an action of the symplectic group $\operatorname{Aut}\left(H_{1}(X, \mathbb{Z})\right) \cong \operatorname{Sp}(2 g, \mathbb{Z})$, which is a quotient of the mapping class group. Moreover, the descended action of $\operatorname{Aut}\left(H_{1}(X, \mathbb{Z})\right)$ on $H^{*}\left(\mathcal{N}_{X}, \mathbb{Q}\right)$ extends to an action of $\operatorname{Aut}\left(H_{1}(X, \mathbb{C})\right) \cong \operatorname{Sp}(2 g, \mathbb{C})$ on $H^{*}\left(\mathcal{N}_{X}, \mathbb{C}\right)$. On the other hand, using the fact that $\mathcal{N}_{X}$ is simply connected, the mapping class group acts naturally on the homotopy groups $\pi_{*}\left(\mathcal{N}_{X}\right)$. Therefore, the mapping class group acts on $\pi_{*}\left(\mathcal{N}_{X}\right) \otimes_{\mathbb{Z}} \mathbb{Q}$. 
Fix a symplectic basis of $H_{1}(X, \mathbb{Z})$. Using this basis $\operatorname{Aut}\left(H_{1}(X, \mathbb{Z})\right)$ (respectively, $\operatorname{Aut}\left(H_{1}(X, \mathbb{C})\right)$ ) gets identified with $\operatorname{Sp}(2 g, \mathbb{Z})$ (respectively, $\operatorname{Sp}(2 g, \mathbb{C}))$.

Our first main result is the following (see Theorem 5.2).

Theorem 1.1. The action of the mapping class group on the rational homotopy groups $\pi_{*}\left(\mathcal{N}_{X}\right) \otimes_{\mathbb{Z}} \mathbb{Q}$ factors through an action of the symplectic group $\operatorname{Sp}(2 g, \mathbb{Z})$. This descended action of $\operatorname{Sp}(2 g, \mathbb{Z})$ extends to an action of $\operatorname{Sp}(2 g, \mathbb{C})$ on $\pi_{*}\left(\mathcal{N}_{X}\right) \otimes_{\mathbb{Z}} \mathbb{C}$.

We shall prove this theorem in Section 5 using the formality of $\mathcal{N}_{X}$ and endowing the minimal model of $\mathcal{N}_{X}$ with an action of $\operatorname{Sp}(2 g, \mathbb{C})$.

In Sections 6 and 7 , we study the $\operatorname{Sp}(2 g, \mathbb{C})$ representations $\pi_{n}\left(\mathcal{N}_{X}\right) \otimes_{\mathbb{Z}} \mathbb{C}$. In the special case of $g=2$, we compute the leading representation for each $n \geq 2$ (Theorem 6.3).

In the general case where $g \geq 2$, we find some non-trivial irreducible $\operatorname{Sp}(2 g, \mathbb{C})$ representations contained in $\pi_{n}\left(\mathcal{N}_{X}\right) \otimes_{\mathbb{Z}} \mathbb{C}$ for each $n \geq 2 g$ (see Theorem 6.3 for the case of $g=2$ and Theorem 7.1 for $g>2$ ). We have the following result.

Theorem 1.2. Take any integer $n$ with $n \geq 2 g$. The $\operatorname{Sp}(2 g, \mathbb{C})$ module $\pi_{n}\left(\mathcal{N}_{X}\right) \otimes_{\mathbb{Z}} \mathbb{C}$ is non-trivial. So the action of $\operatorname{Sp}(2 g, \mathbb{Z})$ on the rational homotopy groups $\pi_{n}\left(\mathcal{N}_{X}\right) \otimes_{\mathbb{Z}} \mathbb{Q}$ is non-trivial, and the action of the mapping class group on $\pi_{n}\left(\mathcal{N}_{X}\right)$ is non-trivial.

A simply connected finite CW complex $Z$ (e.g., a compact one-connected manifold) is said to be rationally elliptic if the total dimension of the rational homotopy groups is finite, or in other words,

$$
\sum_{n \in \mathbb{N}} \operatorname{dim} \pi_{n}(Z) \otimes_{\mathbb{Z}} \mathbb{Q}<\infty .
$$

Otherwise, $Z$ is called rationally hyperbolic (see [6]). If $Z$ is rationally elliptic of dimension $N$, then $\pi_{n}(Z) \otimes_{\mathbb{Z}} \mathbb{Q}=0$ for all $n \geq 2 N$ (equivalently, $\pi_{n}(Z$ ) are torsion for $n \geq 2 N$ ). On the other hand, if $Z$ is rationally hyperbolic of dimension $N$, then

$$
f(k)=\sum_{i=1}^{N-1} \operatorname{dim} \pi_{k+i}(Z) \otimes_{\mathbb{Z}} \mathbb{Q}
$$

grows faster than any polynomial in $k$. This dichotomy is discussed in [6].

A byproduct of Theorem 1.2 is the following corollary. 
Corollary 1.3. The moduli space $\mathcal{N}_{X}$ is rationally hyperbolic for all $g \geq 2$.

\section{The moduli space $\mathcal{N}_{X}$}

Let $X$ be an irreducible smooth complex projective curve of genus $g \geq 2$. Fix a holomorphic line bundle $L_{0}$ over $X$ of degree 1 . Let $\mathcal{N}_{X}$ denote the moduli space of stable vector bundles over $X$ of rank two and $\operatorname{det}(E)=\bigwedge^{2} E=L_{0}$. This moduli space $\mathcal{N}_{X}$ is an irreducible smooth complex projective variety of complex dimension $3 g-3$ (see [19]). In particular, it is a compact connected $C^{\infty}$ (real) manifold of dimension $6 g-6$. The complex structure of $X$ endows $\mathcal{N}_{X}$ with a natural Kähler structure [1].

If we take any holomorphic line bundle $L_{1}$ over $X$ of odd degree $2 d+1$, then there is a holomorphic line bundle $\mu$ over $X$ of degree $d$ such that $L_{1} \cong L_{0} \otimes \mu^{2}$. Therefore, the map defined by $E \longmapsto E \otimes \mu$ is an algebraic isomorphism from $\mathcal{N}_{X}$ to the moduli space of stable vector bundles of rank two over $X$ with determinant $L_{1}$. In particular, the isomorphism class of the variety $\mathcal{N}_{X}$ is independent of the choice of the line bundle $L_{0}$.

The diffeomorphism class of the real manifold $\mathcal{N}_{X}$ is independent of the complex structure of $X$. This can be seen as follows. Fix a point $x_{0} \in X$, and set $X^{\prime}=X \backslash\left\{x_{0}\right\}$ to be the complement. Choosing a point $x^{\prime} \in X^{\prime}$, consider the subset

$$
\operatorname{Hom}^{0}\left(\pi_{1}\left(X^{\prime}, x^{\prime}\right), \mathrm{SU}(2)\right) \subset \operatorname{Hom}\left(\pi_{1}\left(X^{\prime}, x^{\prime}\right), \mathrm{SU}(2)\right)
$$

parametrizing all homomorphisms from the fundamental group $\pi_{1}\left(X^{\prime}, x^{\prime}\right)$ to $\mathrm{SU}(2)$ satisfying the condition that the image of the conjugacy class in $\pi_{1}\left(X^{\prime}, x^{\prime}\right)$ corresponding to the free homotopy class of oriented loops in $X^{\prime}$ around $x_{0}$ (with anticlockwise orientation) is $-\mathrm{Id}$. Let

$$
\frac{\operatorname{Hom}^{0}\left(\pi_{1}\left(X^{\prime}, x^{\prime}\right), \mathrm{SU}(2)\right)}{\mathrm{SU}(2)}
$$

be the quotient space for the adjoint action of $\mathrm{SU}(2)$ on itself. It is easy to see that $\mathcal{R}_{g}$ is a connected compact $C^{\infty}$ manifold of dimension $6 g-$ 6 (see [16]). Given any homomorphism $\rho \in \operatorname{Hom}^{0}\left(\pi_{1}\left(X^{\prime}, x^{\prime}\right), \operatorname{SU}(2)\right)$, the corresponding flat vector bundle over the Riemann surface $X^{\prime}$ extends to $X$ as a holomorphic vector bundle with a logarithmic connection which has residue $-\frac{1}{2}$ Id at $x_{0}$ (see [4]). The underlying holomorphic vector bundle $E_{\rho}$ on $X$ is stable and $\operatorname{det}\left(E_{\rho}\right)=\mathcal{O}_{X}\left(x_{0}\right)$. Sending any $\rho$ to $E_{\rho}$ we obtain a diffeomorphism of $\mathcal{R}_{g}$ with $\mathcal{N}_{X}$ for $L_{0}=\mathcal{O}_{X}\left(x_{0}\right)$ (cf. [14]). Therefore, the 
diffeomorphism class of the real manifold $\mathcal{N}_{X}$ is independent of the complex structure of $X$ (it depends only on the genus of $X$ ).

It is easy to see that the manifold $\mathcal{R}_{g}$ in Equation (2.1) is simply connected [16, Corollary 2]. By [1, Theorem 9.10], the cohomology ring $H^{*}\left(\mathcal{N}_{X}, \mathbb{Z}\right)$ is torsion-free, and by [1, Proposition 9.13], we have $H^{2}\left(\mathcal{N}_{X}\right.$, $\mathbb{Z})=\mathbb{Z}$. Consequently, the variety $\mathcal{N}_{X}$ has a natural polarization. Henceforth, we shall denote by $\alpha$ the natural positive (i.e., ample) generator of $H^{2}\left(\mathcal{N}_{X}, \mathbb{Z}\right)$.

Next we will describe an action of the mapping class group on the cohomology of $\mathcal{N}_{X}$. For that purpose, consider the moduli space $\mathcal{M}_{g}^{1}$ parametrizing all isomorphism classes of one-pointed compact Riemann surfaces $(Y, y)$ of genus $g$ with $\operatorname{Aut}(Y, y)=e$ (i.e., $Y$ does not have any non-trivial automorphism that fixes the marked point $y \in Y)$. This moduli space is a smooth irreducible quasiprojective variety of dimension $3 g-2$ defined over the field $\mathbb{C}$. Given any $(Y, y)$, there is a natural choice of a holomorphic line bundle of degree 1 over $Y$, namely $\mathcal{O}_{Y}(y)$. There is a universal family of Riemann surfaces

$$
p: \mathcal{C}_{g} \longrightarrow \mathcal{M}_{g}^{1}
$$

and a holomorphic section $h: \mathcal{M}_{g}^{1} \longrightarrow \mathcal{C}_{g}$ giving the marked point. Let

$$
P: \tilde{\mathcal{N}} \longrightarrow \mathcal{M}_{g}^{1}
$$

be the family of moduli spaces of stable vector bundles of rank two with fixed determinant corresponding to the family of Riemann surfaces in Equation (2.2). For any one-pointed Riemann surface $\underline{x}=(Y, y) \in \mathcal{M}_{g}^{1}$, the fiber $P^{-1}(\underline{x})$ is the moduli space $\mathcal{N}_{Y}$ parametrizing all stable vector bundles over $Y$ of rank two and determinant $\mathcal{O}_{Y}(y)$.

Fix a base point $\underline{x}_{0}=\left(X, x_{0}\right) \in \mathcal{M}_{g}^{1}$ of the moduli space. Let $G_{\mathbb{Z}}$ (respectively, $G_{\mathbb{C}}$ ) denote the group of all automorphisms of $H^{1}(X, \mathbb{Z})$ (respectively, $H^{1}(X, \mathbb{C})$ ) preserving the symplectic pairing given by the cup product. Choosing a symplectic basis of $H^{1}(X, \mathbb{Z})$, the groups $G_{\mathbb{Z}}$ and $G_{\mathbb{C}}$ get identified with $\operatorname{Sp}(2 g, \mathbb{Z})$ and $\operatorname{Sp}(2 g, \mathbb{C})$ respectively.

Convention. In the sequel, we will interchange $G_{\mathbb{Z}}$ (respectively, $G_{\mathbb{C}}$ ) and $\operatorname{Sp}(2 g, \mathbb{Z})$ (respectively, $\operatorname{Sp}(2 g, \mathbb{C})$ ).

Consider the local system $R^{1} p_{*} \underline{\underline{Z}}$ on $\mathcal{M}_{g}^{1}$, where $p$ is the projection in Equation (2.2), and $\underline{\underline{Z}}$ is the constant local system on $\mathcal{C}_{g}$ with stalk $\mathbb{Z}$. Using its monodromy, the group $G_{\mathbb{Z}}$ is a quotient of the fundamental group

$$
\Gamma_{g}^{1}:=\pi_{1}\left(\mathcal{M}_{g}^{1}, \underline{x}_{0}\right) .
$$


This group $\Gamma_{g}^{1}$ is known as the mapping class group, and the kernel of the projection of $\Gamma_{g}^{1}$ to $G_{\mathbb{Z}}$ is known as the Torelli group.

Actually, the mapping class group has a natural action on the moduli space $\mathcal{N}_{X}=P^{-1}\left(\underline{x}_{0}\right)$. To see this action, note that using the earlier mentioned identification $\mathcal{N}_{X}=\mathcal{R}_{g}$ (defined in Equation (2.1)), the fiber bundle $P$ in Equation (2.3) has a natural flat connection (this flat connection is not holomorphic). The monodromy of this flat connection gives an action of $\Gamma_{g}^{1}=\pi_{1}\left(\mathcal{M}_{g}^{1}, \underline{x}_{0}\right)$ on $\mathcal{N}_{X}$; more details can be found in [2].

The action of $\Gamma_{g}^{1}$ on $H^{i}\left(\mathcal{N}_{X}, \mathbb{Z}\right)$ induced by the above action of $\Gamma_{g}^{1}$ on $\mathcal{N}_{X}$ evidently coincides with the monodromy representation of the local system $R^{i} P_{*} \underline{\mathbb{Z}}$ on $\mathcal{M}_{g}^{1}$, where $\underline{\mathbb{Z}}$ is the constant local system on $\tilde{\mathcal{N}}$ with stalk $\mathbb{Z}$.

Proposition 2.1. The action of the mapping class group on the cohomology algebra $H^{*}\left(\mathcal{N}_{X}, \mathbb{Q}\right)$ factors through an action of the symplectic group $G_{\mathbb{Z}}=$ $\operatorname{Sp}(2 g, \mathbb{Z})$. Moreover, this action of $\operatorname{Sp}(2 g, \mathbb{Z})$ on $H^{i}\left(\mathcal{N}_{X}, \mathbb{Q}\right)$ extends to an action of $G_{\mathbb{C}}=\operatorname{Sp}(2 g, \mathbb{C})$ on $H^{i}\left(\mathcal{N}_{X}, \mathbb{C}\right)$.

Proof. The cohomology algebra $H^{*}\left(\mathcal{N}_{X}, \mathbb{Q}\right)$ is generated by the Künneth components of the second Chern class of the adjoint bundle of a universal vector bundle over $X \times \mathcal{N}_{X}$ (see $[1,18]$ and also Section 3 ). Note that although there is no unique universal bundle over $X \times \mathcal{N}_{X}$, any two universal bundles differ by tensoring with a line bundle pulled back from $\mathcal{N}_{X}$. Therefore, the universal adjoint bundle is unique. Consequently, the local system $\bigoplus_{i \geq 0} R^{i} P_{*} \underline{\mathbb{C}}$ on $\mathcal{M}_{g}^{1}$, where $\underline{\mathbb{C}}$ is the constant local system on $\widetilde{\mathcal{N}}$ with stalk $\mathbb{C}$, is a quotient of some local system on $\mathcal{M}_{g}^{1}$ of the form

$$
\mathcal{W}:=\bigoplus_{j=1}^{\ell}\left(\left(\bigoplus_{i=0}^{2} R^{i} p_{*} \underline{\underline{\mathbb{C}}}\right)^{\oplus a_{j}}\right)^{\otimes b_{j}}
$$

where $\ell, a_{j}, b_{j} \in \mathbb{N}$, the map $p$ is the projection in Equation (2.2) and $\mathbb{E}$ is the constant local system on $\mathcal{C}_{g}$ with stalk $\mathbb{C}$. In other words, we have a surjective homomorphism of local systems

$$
\mathcal{W} \longrightarrow \bigoplus_{i \geq 0} R^{i} P_{*} \underline{\mathbb{C}} \longrightarrow 0
$$

Both $R^{0} p_{*} \underline{\underline{C}}$ and $R^{2} p_{*} \underline{\underline{C}}$ are constant local systems on $\mathcal{M}_{g}^{1}$, and the monodromy of the local system $R^{1} p_{*} \underline{\underline{C}}$, by definition, factors through $G_{\mathbb{Z}}$. Consequently, the monodromy representation

$$
\Gamma_{g}^{1} \longrightarrow \operatorname{Aut}\left(\mathcal{W}_{\underline{x}_{0}}\right)
$$


of the mapping class group for the local system $\mathcal{W}$ on $\mathcal{M}_{g}^{1}$ factors through $G_{\mathbb{Z}}$. Hence, the Torelli group is in the kernel of the monodromy representation

$$
\Gamma_{g}^{1} \longrightarrow \prod_{i \geq 0} \operatorname{Aut}\left(\left(R^{i} P_{*} \underline{\mathbb{C}}\right)_{\underline{x}_{0}}\right)
$$

of the mapping class group for the quotient local system in Equation (2.4). Therefore, the homomorphism in Equation (2.6) factors through the quotient $G_{\mathbb{Z}}$ of $\Gamma_{g}^{1}$.

To prove that the action of $\operatorname{Sp}(2 g, \mathbb{Z})$ on $H^{i}\left(\mathcal{N}_{X}, \mathbb{Q}\right)$ extends to an action of $G_{\mathbb{C}}=\operatorname{Sp}(2 g, \mathbb{C})$ on $H^{i}\left(\mathcal{N}_{X}, \mathbb{C}\right)$, first note that the monodromy representation

$$
G_{\mathbb{Z}} \longrightarrow \operatorname{Aut}\left(\mathcal{W}_{\underline{x}_{0}}\right)
$$

in Equation (2.5) extends to a homomorphism from $\operatorname{Sp}(2 g, \mathbb{C})$. The kernel of the surjective homomorphism

$$
\mathcal{W}_{\underline{x}_{0}} \longrightarrow \bigoplus_{i \geq 0}\left(R^{i} P_{*} \underline{\mathbb{C}}\right)_{\underline{x}_{0}}
$$

obtained from Equation (2.4) is preserved by $G_{\mathbb{Z}}$. On the other hand, $\operatorname{Sp}(2 g, \mathbb{Z})$ is Zariski dense in $\operatorname{Sp}(2 g, \mathbb{C})$ (see [3]). Hence the kernel of the homomorphism in Equation (2.7) is preserved by the action of $\operatorname{Sp}(2 g, \mathbb{C})$ on $\mathcal{W}_{\underline{x}_{0}}$. Consequently, the action of $\operatorname{Sp}(2 g, \mathbb{C})$ on $\mathcal{W}_{\underline{x}_{0}}$ induces an action of $\operatorname{Sp}(2 g, \mathbb{C})$ on the quotient in Equation $(2.7)$. This completes the proof of the proposition.

\section{Cohomology ring of $\mathcal{N}_{X}$}

Let us recall the known description of the cohomology $\operatorname{ring} H^{*}\left(\mathcal{N}_{X}, \mathbb{Q}\right)$ of the moduli space $\mathcal{N}_{X}$ (see $\left.[9,11,20]\right)$. Consider a universal bundle $\mathcal{U} \rightarrow$ $X \times \mathcal{N}_{X}$. Let $\operatorname{End}_{0}(\mathcal{U}) \rightarrow X \times \mathcal{N}_{X}$ be the adjoint vector bundle (we recall that $\operatorname{End}_{0}(\mathcal{U}) \subset \operatorname{End}(\mathcal{U})$ is subbundle of rank three given by the trace-free endomorphisms of the fibers of $\mathcal{U})$. The Künneth decomposition of the second Chern class $c_{2}\left(\operatorname{End}_{0}(\mathcal{U})\right) \in H^{4}\left(X \times \mathcal{N}_{X}, \mathbb{Z}\right)$ can be written as

$$
c_{2}\left(\operatorname{End}_{0}(\mathcal{U})\right)=2[X] \otimes \alpha+4 \psi-1 \otimes \beta,
$$

where $\beta \in H^{4}\left(\mathcal{N}_{X}, \mathbb{Z}\right),[X] \in H^{2}(X, \mathbb{Z})$ denotes the fundamental class of the Riemann surface $X, \alpha \in H^{2}\left(\mathcal{N}_{X}, \mathbb{Z}\right)$ as before is the positive generator of $H^{2}\left(\mathcal{N}_{X}, \mathbb{Z}\right)=\mathbb{Z}$, and $\psi \in H^{1}(X, \mathbb{Z}) \otimes_{\mathbb{Z}} H^{3}\left(\mathcal{N}_{X}, \mathbb{Z}\right)$. Let $\left\{c_{1}, \ldots, c_{2 g}\right\}$ 
be a symplectic basis of $H^{1}(X, \mathbb{Z})$, which means that $c_{i} \cup c_{i+g}=[X]$ for all $1 \leq i \leq g$, and $c_{j} \cup c_{k}=0$ for all $j, k$ with $|j-k| \neq g$. It is known that $\psi=\sum_{i=1}^{2 g} c_{i} \otimes \gamma_{i}$, where $\left\{\gamma_{1}, \ldots, \gamma_{2 g}\right\}$ is a basis for $H^{3}\left(\mathcal{N}_{X}, \mathbb{Z}\right)$; see [12]. In other words, $\psi$ gives an isomorphism

$$
H^{1}(X, \mathbb{Z})=H^{1}(X, \mathbb{Z})^{*} \longrightarrow H^{3}(\mathcal{N}, \mathbb{Z})
$$

The elements $\alpha, \beta$ and $\gamma_{i}, 1 \leq i \leq 2 g$, together generate $H^{*}\left(\mathcal{N}_{X}, \mathbb{Q}\right)$ as an algebra $[18,1,21]$. We can rephrase this as saying that there exists an epimorphism

$$
F: \bigwedge\left(\alpha, \gamma_{1}, \ldots, \gamma_{2 g}, \beta\right):=\mathbb{Q}[\alpha, \beta] \otimes \wedge\left(\gamma_{1}, \ldots, \gamma_{2 g}\right) \rightarrow H^{*}\left(\mathcal{N}_{X}, \mathbb{Q}\right)
$$

where $\operatorname{deg}(\alpha)=2, \operatorname{deg}(\beta)=4$ and $\operatorname{deg}\left(\gamma_{i}\right)=3,1 \leq i \leq 2 g$. Here $\bigwedge$ means the free graded algebra generated by the given elements, which is the tensor product of the symmetric algebra on the even-degree elements and the exterior algebra on the odd-degree elements.

We shall denote by $W$ the standard $\mathbb{Q}$ representation of $G_{\mathbb{Z}}=$ $\operatorname{Sp}(2 g, \mathbb{Z})$, so

$$
H^{1}(X, \mathbb{Q}) \cong W
$$

We noted in Section 2 that the monodromy action of $\Gamma_{g}^{1}$ on $H^{*}\left(\mathcal{N}_{X}, \mathbb{Q}\right)$ factors through an action of $\operatorname{Sp}(2 g, \mathbb{Z})$. It is easy to see that this action fixes both $\alpha$ and $\beta$, and furthermore, the isomorphism in Equation (3.2) is $\operatorname{Sp}(2 g, \mathbb{Z})$-equivariant. Therefore,

$$
H^{3}\left(\mathcal{N}_{X}, \mathbb{Q}\right) \cong H^{1}(X, \mathbb{Q})^{*} \cong W^{*} \cong W
$$

as $\operatorname{Sp}(2 g, \mathbb{Z})$ representations.

Let

$$
H_{I}^{*}\left(\mathcal{N}_{X}, \mathbb{Q}\right) \subset H^{*}\left(\mathcal{N}_{X}, \mathbb{Q}\right)
$$

be the subalgebra fixed pointwise by the action of $\operatorname{Sp}(2 g, \mathbb{Z})$. The epimorphism in Equation (3.3) is $\operatorname{Sp}(2 g, \mathbb{Z})$-equivariant, and $\operatorname{Sp}(2 g, \mathbb{Z})$ is Zariski dense in the reductive group $\operatorname{Sp}(2 g, \mathbb{C})[3]$. Using these we conclude that the invariant part $H_{I}^{*}\left(\mathcal{N}_{X}, \mathbb{Q}\right)$ is generated by $\alpha, \beta$ and $\gamma=-2 \sum_{i=1}^{g} \gamma_{i} \gamma_{i+g}$ (the factor of -2 is for convenience, to be in accordance with the existing literature). Then the epimorphism $F$ in Equation (3.3) gives an epimorphism

$$
\mathbb{Q}[\alpha, \beta, \gamma] \rightarrow H_{I}^{*}\left(\mathcal{N}_{X}, \mathbb{Q}\right)
$$


where $\operatorname{deg}(\alpha)=2, \operatorname{deg}(\beta)=4$ and $\operatorname{deg}(\gamma)=6$. Hence we may write

$$
H_{I}^{*}\left(\mathcal{N}_{X}, \mathbb{Q}\right)=\frac{\mathbb{Q}[\alpha, \beta, \gamma]}{I_{g}},
$$

where $I_{g}$ is an ideal of relations satisfied by $\alpha, \beta$ and $\gamma$.

For each $0 \leq k \leq g$, the primitive component of $\wedge^{k} W$ is defined as

$$
\wedge_{0}^{k} W=\operatorname{ker}\left(\gamma^{g-k+1}: \wedge^{k} W \longrightarrow \wedge^{2 g-k+2} W\right) .
$$

The spaces $\wedge_{0}^{k} W$ are irreducible $\operatorname{Sp}(2 g, \mathbb{Z})$ representations.

The descriptions of the ideal $I_{g}$ and the cohomology ring $H^{*}\left(\mathcal{N}_{X}, \mathbb{Q}\right)$ are given in the following proposition.

Proposition 3.1 $[9,20]$. Define $q_{0}^{1}=1, q_{0}^{2}=0, q_{0}^{3}=0$ and then recursively, for all $r \geq 1$,

$$
\begin{aligned}
& q_{r+1}^{1}=\alpha q_{r}^{1}+r^{2} q_{r}^{2}, \\
& q_{r+1}^{2}=\beta q_{r}^{1}+\frac{2 r}{r+1} q_{r}^{3}, \\
& q_{r+1}^{3}=\gamma q_{r}^{1} .
\end{aligned}
$$

Then $I_{g}=\left(q_{g}^{1}, q_{g}^{2}, q_{g}^{3}\right) \subset \mathbb{Q}[\alpha, \beta, \gamma]$, for all $g \geq 1$. Note that $\operatorname{deg}\left(q_{g}^{1}\right)=2 g$, $\operatorname{deg}\left(q_{g}^{2}\right)=2 g+2$ and $\operatorname{deg}\left(q_{g}^{3}\right)=2 g+4$. Moreover the $\operatorname{Sp}(2 g, \mathbb{Z})$ decomposition of $H^{*}\left(\mathcal{N}_{X}, \mathbb{Q}\right)$ is

$$
H^{*}\left(\mathcal{N}_{X}, \mathbb{Q}\right)=\bigoplus_{k=0}^{g-1} \wedge_{0}^{k} W \otimes \frac{\mathbb{Q}[\alpha, \beta, \gamma]}{I_{g-k}}
$$

Lemma 3.2. The vector space

$$
E=\left\langle q_{g}^{1}\right\rangle \oplus\left\langle q_{g}^{2}\right\rangle \oplus\left(q_{g-1}^{1} \cdot W\right) \oplus\left(q_{g-2}^{1} \cdot \wedge_{0}^{2} W\right) \oplus \cdots \oplus\left(q_{1}^{1} \cdot \wedge_{0}^{g-1} W\right) \oplus \wedge_{0}^{g} W
$$

realized as a subspace of $\mathbb{A}:=\bigwedge\left(\alpha, \gamma_{1}, \ldots, \gamma_{2 g}, \beta\right)=\mathbb{Q}[\alpha, \beta] \otimes \wedge\left(\gamma_{1}, \ldots, \gamma_{2 g}\right)$ using the identification $W=\left\langle\gamma_{1}, \ldots, \gamma_{2} g\right\rangle$, generates the ideal $\operatorname{kernel}(F)$ of the map $F$ in Equation (3.3).

Proof. Clearly we have $E \subset \operatorname{kernel}(F)$. We will prove the reverse inclusion

$$
\operatorname{kernel}(F) \subset I(E) \text {, }
$$

where $I(E)$ is the ideal generated by $E$ in $\mathbb{A}$. 
By Proposition 3.1, $\operatorname{kernel}(F)$ is generated by $q_{g-k}^{i} \cdot \wedge_{0}^{k} W$, where $i \in$ $[1,3]$ and $k \in[0, g]$. Note that since $q_{0}^{2}=0$ and $q_{0}^{3}=0$, it suffices to prove the following two:

(1) $q_{g-k}^{2} \cdot \wedge_{0}^{k} W \subset I(E)$ for $1 \leq k \leq g-1$, and

(2) $q_{g-k}^{3} \cdot \wedge_{0}^{k} W \subset I(E)$ for $0 \leq k \leq g-1$.

We shall use the following inclusions:

$$
\begin{aligned}
& \gamma \cdot \wedge_{0}^{j} W \subset I\left(\wedge_{0}^{j+1} W\right), \quad 0 \leq j \leq g-1, \\
& \wedge_{0}^{j+1} W \subset I\left(\wedge_{0}^{j} W\right), \quad 0 \leq j \leq g-1 .
\end{aligned}
$$

For proving Equation (3.7), first note that $\wedge_{0}^{j} W$ is an irreducible $\operatorname{Sp}(2 g, \mathbb{Z})$ representation, so it is enough to see that there is a non-zero element in $\gamma \cdot \wedge_{0}^{j} W$ which lies in $I\left(\wedge_{0}^{j+1} W\right)$. Consider $\gamma_{1} \cdots \gamma_{j} \in \wedge_{0}^{j} W$. Then

$$
\gamma \cdot \gamma_{1} \cdots \gamma_{j}=-2 \sum_{i=j+1}^{g} \gamma_{1} \cdots \gamma_{j} \gamma_{j+1} \gamma_{j+1+g}
$$

and $\gamma_{1} \cdots \gamma_{j+1} \in \wedge_{0}^{j+1} W$. Therefore $\gamma \cdot \gamma_{1} \cdots \gamma_{j} \in I\left(\wedge_{0}^{j+1} W\right)$, as required.

To prove Equation (3.8), we first note that $\wedge_{0}^{j+1} W$ is an irreducible $\operatorname{Sp}(2 g, \mathbb{Z})$ representation and $\gamma_{1} \cdots \gamma_{j+1} \in \wedge_{0}^{j+1} W$. Clearly we have $\gamma_{1} \cdots \gamma_{j}$ $\in \wedge_{0}^{j} W$. Hence it follows that $\gamma_{1} \cdots \gamma_{j} \gamma_{j+1} \in I\left(\wedge_{0}^{j} W\right)$. This gives the required inclusion.

Using Equation (3.7), we have that

$$
q_{g-k}^{3} \cdot \wedge_{0}^{k} W=q_{g-k-1}^{1} \gamma \cdot \wedge_{0}^{k} W \subset I\left(q_{g-k-1}^{1} \cdot \wedge_{0}^{k+1} W\right) \subset I(E)
$$

for all $0 \leq k \leq g-1$. Also, using Equation (3.8) we have

$$
\begin{aligned}
q_{g-k}^{2} \cdot \wedge_{0}^{k} W & =\frac{1}{(g-k)^{2}}\left(q_{g-k+1}^{1}-\alpha q_{g-k}^{1}\right) \cdot \wedge_{0}^{k} W \\
& \subset I\left(q_{g-k+1}^{1} \cdot \wedge_{0}^{k-1} W \oplus q_{g-k}^{1} \cdot \wedge_{0}^{k} W\right) \subset I(E)
\end{aligned}
$$

for all $1 \leq k \leq g-1$

Remark 3.3. The subspace $E$ in Lemma 3.2 is minimal in the sense that no proper subspace of $E$ generates $\operatorname{kernel}(F)$. 


\section{Minimal models}

Let us recall some definitions and results about minimal models (see [5, 8] for more details). Let $(A, d)$ be a differential graded algebra (in the sequel, we shall just say a differential algebra). This means that $A$ is a graded (in nonnegative degrees) commutative algebra over a field $K$, of characteristic zero, and $d: A^{n} \longrightarrow A^{n+1}$ is a differential which satisfies the derivation condition which says that

$$
d(a \cdot b)=(d a) \cdot b+(-1)^{\operatorname{deg}(a)} a \cdot(d b)
$$

where $\operatorname{deg}(a)$ is the degree of $a$. Throughout this article we shall assume that $K=\mathbb{C}$, the field of complex numbers.

Morphisms between differential algebras are required to be degreepreserving algebra maps that commute with the differentials. Given a differential algebra $(A, d)$, we denote by $H^{*}(A, d)$ its cohomology. We say that $A$ is connected if $H^{0}(A, d)=\mathbb{C}$, and one-connected if, in addition, $H^{1}(A, d)=0$.

A differential algebra $(A, d)$ is said to be minimal if the following two hold:

(i) $A$ is free as a graded algebra, that is, $A=\bigwedge V$, where $V=\oplus_{i>0} V^{i}$ is a graded vector space, and

(ii) there exists a collection of generators $\left\{a_{\tau}\right\}_{\tau \in I}$ of the algebra $A$, where $I$ is some well-ordered index set, $\operatorname{such}$ that $\operatorname{deg}\left(a_{\mu}\right) \leq \operatorname{deg}\left(a_{\tau}\right)$ if $\mu<\tau$ and each $d a_{\tau}$ is expressed in terms of preceding $a_{\mu}, \mu<\tau$.

As before, $\bigwedge V$ is the tensor product of the symmetric algebra on the even degree part of $V$ with the exterior algebra on the odd degree part of $V$

For notational convenience, we shall use the dot "." to denote the product operation on $\bigwedge V$.

For any $n$, define $V^{\leq n}:=\bigoplus_{i \leq n} V^{i}$. So $\bigwedge V^{\leq n}=\bigwedge\left(\bigoplus_{i \leq n} V^{i}\right)$ is the subalgebra generated by elements of degrees at most $n$. For any $m$, let $(\bigwedge V)^{m}$ denote the subspace of $\bigwedge V$ spanned by all elements of total degree $m$. Finally, for $k \geq 1$, let $\bigwedge^{\geq k} V$ denote the ideal formed by elements which are products of at least $k$ generators. In other words,

$$
\bigwedge^{+} V:=(\bigwedge V)^{>0}=\bigoplus_{m>0}(\bigwedge V)^{m}
$$


and

$$
\bigwedge^{\geq k}=\overbrace{\bigwedge^{+} V \cdots \bigwedge^{+} V}^{k \text {-times }} .
$$

Note that the condition (ii) in the definition of minimality implies that $d: V \longrightarrow \bigwedge^{\geq 2} V$, and hence $d: \bigwedge^{i} V \longrightarrow \bigwedge^{\geq(i+1)} V$, for all $i \geq 1$. Notations like $\left(\bigwedge^{\geq i} V^{<j}\right)^{n}$ have natural meaning.

Given a differential algebra $(A, d)$, we shall say that $(\bigwedge V, d)$ is a $\min$ imal model of $(A, d)$ if $(\bigwedge V, d)$ is minimal and there exists a morphism of differential graded algebras $\rho:(\bigwedge V, d) \longrightarrow(A, d)$ such that the induced homomorphism of cohomologies

$$
\rho^{*}: H^{*}(\bigwedge V, d) \longrightarrow H^{*}(A, d)
$$

is an isomorphism. Such a homomorphism $\rho$ is called a quasi-isomorphism. Any one-connected differential algebra $(A, d)$ has a minimal model unique up to an isomorphism $[5,8]$.

A minimal model of a connected differentiable manifold $M$ is a minimal model $(\bigwedge V, d)$ for the de Rham complex $\left(\Omega^{*}(M, \mathbb{C}), d\right)$ of complex $C^{\infty}$ differential forms on $M$. If $M$ is simply connected, then the dual of the complex homotopy vector space $\pi_{i}(M) \otimes_{\mathbb{Z}} \mathbb{C}$ is isomorphic to $V^{i}$ for any $i>0$ (see [8]).

A minimal model $(\bigwedge V, d)$ is said to be formal if there is a morphism of differential algebras

$$
\psi:(\bigwedge V, d) \longrightarrow\left(H^{*}(\bigwedge V, d), 0\right)
$$

which induces the identity map on cohomology. This means that $(\bigwedge V, d)$ is the minimal model of the algebra $\left(H^{*}(\bigwedge V, d), 0\right)$ with zero differential.

We shall say that a connected differentiable manifold $M$ is formal if its minimal model is formal, or equivalently, the two differential algebras $\left(\Omega^{*}(M, \mathbb{C}), d\right)$ and $\left(H^{*}(M, \mathbb{C}), 0\right)$ have the same minimal model. Therefore, if $M$ is formal and simply connected, then the complex homotopy groups $\pi_{i}(M) \otimes_{\mathbb{Z}} \mathbb{C}$ are obtained by computing the minimal model of $\left(H^{*}(M, \mathbb{C}), 0\right)$.

The main result of [5] gives the following strong topological restriction on the rational homotopy type of Kähler manifolds.

Theorem 4.1 [5]. Let $M$ be a compact connected Kähler manifold. Then $M$ is formal. 
Therefore, the minimal model of a compact connected Kähler manifold $M$ can be obtained from the minimal model of its cohomology algebra $\left(H^{*}(M, \mathbb{C}), 0\right)$. Moreover, if the Kähler manifold $M$ is simply connected, this process will also give us the complex homotopy group $\pi_{i}(M) \otimes_{\mathbb{Z}} \mathbb{C}$ of $M$.

We will briefly review a construction of the minimal model of a differential algebra $(A, d)$. For simplicity, we shall assume that $(A, d)$ is oneconnected. We need to find a graded vector space $V=\oplus_{n \geq 1} V^{n}$, a differential $d$, with

$$
\left.d\right|_{V^{n}}: V^{n} \longrightarrow \bigwedge^{\geq 2} V^{\leq(n-1)},
$$

and a graded linear map

$$
\rho=\sum \rho_{n}: V=\oplus V^{n} \longrightarrow A=\oplus A^{n}
$$

such that the induced homomorphism $\rho: \bigwedge V \longrightarrow A$ respects the differentials, which means that $\rho \circ d=d \circ \rho$, and furthermore, the map on cohomology

$$
\rho^{*}: H^{*}(\bigwedge V, d) \longrightarrow H^{*}(A, d)
$$

is an isomorphism.

We shall construct $V^{n}, \rho_{n}$ and $\left.d\right|_{V^{n}}$, where $n \geq 1$, using induction on $n$. They will satisfy the following conditions:

(i) $\rho_{n}: V^{n} \longrightarrow A^{n}$;

(ii) $d_{n}=\left.d\right|_{V^{n}}: V^{n} \longrightarrow \bigwedge^{\geq 2} V^{\leq(n-1)}$;

(iii) $\rho_{\leq(n-1)} \circ d_{n}=d \circ \rho_{n}$ on $V^{n}$, where $\rho_{\leq(n-1)}: \bigwedge V^{\leq(n-1)} \longrightarrow A$ is induced by the map $\rho_{i}, i \leq n-1$;

(iv) $\rho_{\leq n}^{*}: H^{i}\left(\bigwedge V^{\leq n}, d\right) \stackrel{\simeq}{\longrightarrow} H^{i}(A, d)$ is an isomorphism for $i \leq n$;

(v) $\rho_{\leq n}^{*}: H^{n+1}\left(\bigwedge V^{\leq n}, d\right) \hookrightarrow H^{n+1}(A, d)$ is an injection.

From these conditions it follows that the map $\rho:(\bigwedge V, d) \longrightarrow(A, d)$, constructed using $\rho_{n}$ on each subspace $V^{n}$, is a quasi-isomorphism. Given any $i$, we evidently have $(\bigwedge V)^{k}=(\bigwedge V \leq(i+1))^{k}$ for all $k \leq i+1$. So $H^{i}(\bigwedge V, d) \cong$ $H^{i}\left(\bigwedge V^{\leq(i+1)}, d\right)$. The composition

$$
\left(\bigwedge V^{\leq(i+1)}, d\right) \hookrightarrow(\bigwedge V, d) \stackrel{\rho}{\longrightarrow}(A, d)
$$


equals $\rho_{\leq(i+1)}$. Hence

$$
\rho^{*}=\rho_{\leq(i+1)}^{*}: H^{i}(\bigwedge V, d) \cong H^{i}\left(\bigwedge V^{\leq(i+1)}, d\right) \stackrel{\simeq}{\longrightarrow} H^{i}(A, d)
$$

is an isomorphism. This proves that $(\bigwedge V, d)$ is a minimal model for $(A, d)$.

To construct $V^{n}, \rho_{n}$ and $\left.d\right|_{V^{n}}$, we start with $V^{1}=0$. All conditions (i)-(v) hold trivially, since $H^{1}(A, d)=0$.

Now assume that conditions $(\mathrm{i})-(\mathrm{v})$ are satisfied for all $j \in[1, n-1]$ with $n-1 \geq 1$; let us see that we can find $V^{n}, \rho_{n}$ and $d_{n}$ also fulfilling these conditions. Take

$$
\begin{aligned}
& V^{n}=C^{n} \oplus N^{n}, \\
& C^{n}=\operatorname{coker}\left(\rho_{\leq(n-1)}^{*}: H^{n}\left(\bigwedge V^{\leq(n-1)}, d\right) \hookrightarrow H^{n}(A, d)\right), \\
& N^{n}=\operatorname{ker}\left(\rho_{\leq(n-1)}^{*}: H^{n+1}\left(\bigwedge V^{\leq(n-1)}, d\right) \longrightarrow H^{n+1}(A, d)\right) .
\end{aligned}
$$

Define $\rho_{n}: V^{n} \longrightarrow A^{n}$ as follows. First, we introduce the notation

$$
\begin{aligned}
& Z^{n}(A, d)=\operatorname{ker}\left(d: A^{n} \longrightarrow A^{n+1}\right) \\
& B^{n}(A, d)=\operatorname{im}\left(d: A^{n-1} \longrightarrow A^{n}\right)
\end{aligned}
$$

for the spaces of cocycles and coboundaries, respectively. Let $\imath_{1}: C^{n} \longrightarrow$ $H^{n}(A, d)$ be a linear map which is a splitting of the projection $H^{n}(A, d) \rightarrow$ $C^{n}$. Also, let $\imath_{2}: H^{n}(A, d) \longrightarrow Z^{n}(A, d)$ be a splitting of the projection $Z^{n}(A, d) \rightarrow H^{n}(A, d)$. Let $\imath_{3}: Z^{n}(A, d) \hookrightarrow A^{n}$ be the inclusion map. Then define

$$
\left.\rho_{n}\right|_{C^{n}}=\imath_{3} \circ \imath_{2} \circ \imath_{1} .
$$

To define $\left.\rho_{n}\right|_{N^{n}}$, let $\jmath_{1}: N^{n} \hookrightarrow H^{n+1}\left(\bigwedge V^{\leq(n-1)}, d\right)$ be the inclusion. Take a splitting $\jmath_{2}: H^{n+1}\left(\bigwedge V^{\leq(n-1)}, d\right) \longrightarrow Z^{n+1}\left(\bigwedge V^{\leq(n-1)}, d\right)$ of the obvious projection. Then $\rho_{\leq(n-1)} \circ \jmath_{2} \circ \jmath_{1}$ has image in $B^{n+1}(A, d) \subset A^{n+1}$. Take a splitting of the map $d: A^{n} \rightarrow B^{n+1}(A, d)$, say

$$
\varrho: B^{n+1}(A, d) \longrightarrow A^{n}
$$

and finally define

$$
\left.\rho_{n}\right|_{N^{n}}=\varrho \circ \rho_{\leq(n-1)} \circ \jmath_{2} \circ \jmath_{1} .
$$


Now, define $d_{n}$ as follows. On $C^{n}$, we set $\left.d_{n}\right|_{C^{n}}=0$. On $N^{n}$, we put $\left.d_{n}\right|_{N^{n}}=\jmath_{3} \circ \jmath_{2} \circ \jmath_{1}$, where

$$
\jmath_{3}: Z^{n+1}\left(\bigwedge^{\leq(n-1)}, d\right) \hookrightarrow\left(\bigwedge^{\leq(n-1)}\right)^{n+1}=\left(\bigwedge^{\geq 2} V^{\leq(n-1)}\right)^{n+1}
$$

is the inclusion. Clearly condition (ii) holds.

To check condition (iii), we need to verify that $\rho_{\leq(n-1)} \circ d_{n}=d \circ \rho_{n}$. On $C^{n}$, we have $\rho_{\leq(n-1)} \circ d_{n}=0$ and $d \circ \rho_{n}=d \circ \imath_{3} \circ \imath_{2} \circ \imath_{1}=0$. On $N^{n}$, we have

$$
\rho_{\leq(n-1)} \circ d_{n}=\rho_{\leq(n-1)} \circ \jmath_{3} \circ \jmath_{2} \circ \jmath_{1}=d \circ \varrho \circ \rho_{\leq(n-1)} \circ \jmath_{2} \circ \jmath_{1}=d \circ \rho_{n}
$$

as $d \circ \varrho=$ Id. Therefore, condition (iii) holds.

Consider the inclusion $j:\left(\bigwedge V^{\leq(n-1)}, d\right) \hookrightarrow\left(\bigwedge V^{\leq n}, d\right)$ and the cokernel

$$
B=\left(\bigwedge V^{\leq n}\right) /\left(\bigwedge V^{\leq(n-1)}\right)
$$

Then $(B, d)$ is a graded differential algebra, and $B^{i}=0$ for all $i<n$, and also, $B^{n}=V^{n}=C^{n} \oplus N^{n}$. We have $\left(\bigwedge V^{\leq n}\right)^{n+1}=\left(\bigwedge V^{\leq(n-1)}\right)^{n+1}$ as $V^{1}=$ 0 , and hence $B^{n+1}=0$. Therefore,

$$
j^{*}: H^{k}\left(\bigwedge V^{\leq(n-1)}, d\right) \longrightarrow H^{k}\left(\bigwedge^{\leq n}, d\right)
$$

is an isomorphism for all $k<n$. As $\rho_{\leq n}^{*} \circ j^{*}=\rho_{\leq(n-1)}^{*}$, we have that

$$
\rho_{\leq n}^{*}: H^{k}\left(\bigwedge V^{\leq n}, d\right) \longrightarrow H^{k}(A, d)
$$

is an isomorphism for all $k<n$.

To deal with the cases where $k=n, n+1$, consider the long-exact sequence associated to $\bigwedge V^{\leq(n-1)} \hookrightarrow \bigwedge^{\leq n} \longrightarrow B$,

$$
\begin{aligned}
& 0 \rightarrow H^{n}\left(\bigwedge V^{\leq(n-1)}, d\right) \quad \stackrel{j^{*}}{\rightarrow} H^{n}\left(\bigwedge V^{\leq n}, d\right) \quad \begin{array}{ll}
\rightarrow & H^{n}(B, d)=B^{n} \\
& =C^{n} \oplus N^{n}
\end{array} \\
& \stackrel{\partial^{*}}{\longrightarrow} H^{n+1}\left(\bigwedge V^{\leq(n-1)}, d\right) \rightarrow H^{n+1}\left(\bigwedge V^{\leq n}, d\right) \rightarrow
\end{aligned}
$$

For $\quad x=u+w \in B^{n}=C^{n} \oplus N^{n}$, we have $\partial^{*}(x)=[d(u+w)]=\left[\jmath_{3} \circ \jmath_{2} \circ\right.$ $\left.\jmath_{1}(w)\right]=\jmath_{1}(w)$. 
Therefore the exact sequence Equation (4.1) splits into two short exact sequences:

$$
0 \longrightarrow H^{n}\left(\bigwedge^{\leq(n-1)}, d\right) \longrightarrow H^{n}\left(\bigwedge^{\leq n}, d\right) \longrightarrow C^{n} \longrightarrow 0
$$

and

$$
0 \longrightarrow N^{n} \stackrel{\jmath_{1}}{\longrightarrow} H^{n+1}\left(\bigwedge^{\leq(n-1)}, d\right) \longrightarrow H^{n+1}\left(\bigwedge^{\leq n}, d\right) \longrightarrow 0
$$

From Equation (4.2), we have

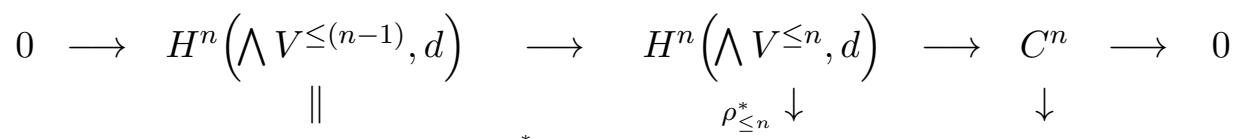

$$
\begin{aligned}
& 0 \longrightarrow H^{n}\left(\bigwedge V^{\leq(n-1)}, d\right) \stackrel{\rho_{\leq(n-1)}^{*}}{\longrightarrow} H^{n}(A, d) \quad \longrightarrow C^{n} \longrightarrow 0 \text {. }
\end{aligned}
$$

We note that the right vertical arrow is the identity map. Indeed, it sends $u \in C^{n}$ to the class of $\rho_{n}(u)=\imath_{3}\left(\imath_{2}\left(\imath_{1}(u)\right)\right)$ in

$$
\operatorname{coker}\left(\rho_{\leq(n-1)}^{*}: H^{n}\left(\bigwedge V^{\leq(n-1)}, d\right) \longrightarrow H^{n}(A, d)\right),
$$

which is $u$ itself. Thus the middle vertical arrow is an isomorphism, proving condition (iv) for $k=n$.

In Equation (4.3), the homomorphism $\jmath_{1}$ is the inclusion of

$$
N^{n}=\operatorname{ker}\left(\rho_{\leq(n-1)}^{*}: H^{n+1}\left(\bigwedge V^{\leq(n-1)}, d\right) \longrightarrow H^{n+1}(A, d)\right)
$$

in $H^{n+1}\left(\bigwedge V^{\leq(n-1)}, d\right)$. So $\rho_{\leq(n-1)}^{*}$ induces an inclusion

$$
H^{n+1} \frac{\left(\bigwedge V^{\leq(n-1)}, d\right)}{N^{n}} \cong H^{n+1}\left(\bigwedge V^{\leq n}, d\right) \hookrightarrow H^{n+1}(A, d) \text {. }
$$

This map actually coincides with $\rho_{\leq n}^{*}$, since $\left(\bigwedge V^{\leq(n-1)}\right)^{n+1}=\left(\bigwedge V^{\leq n}\right)^{n+1}$. This proves that condition (v) holds.

Remark 4.2. Note that $d: N^{n} \longrightarrow(\bigwedge V)^{n+1}$ is always injective, and $\left.d\right|_{C^{n}}=0$ for all $n$.

Remark 4.3. If $(A, 0)$ is a differential algebra with zero differential, then the minimal model $\rho:(\bigwedge V, d) \longrightarrow(A, 0)$ constructed before has the property that $\rho\left(N^{n}\right)=0$ for all $n$. 


\section{Minimal models and $G$-actions}

Let $G$ be a reductive complex Lie group. An action of the group $G$ on a differential algebra $(A, d)$ is a representation $r: G \longrightarrow \mathrm{GL}(A)$ such that

- $r(z)\left(A^{n}\right)=A^{n}$ for all $n \geq 0$ and $z \in G$,

- $r(z)\left(v_{1} \cdot v_{2}\right)=r(z)\left(v_{1}\right) \cdot r(z)\left(v_{2}\right)$ for all $v_{1}, v_{2} \in A$ and

- $r(z)(d v)=d(r(z)(v))$ for all $z \in G$ and $v \in A$.

If $G$ acts on $(A, d)$, then we say that $(A, d)$ is a $G$-differential algebra.

A $G$-minimal differential algebra is a minimal differential algebra $(\bigwedge V, d)$ on which $G$ acts satisfying the condition that each graded vector space $V^{n}$, $n \geq 0$, is preserved by the action of $G$. A $G$-minimal model of a $G$-differential algebra $(A, d)$ is a $G$-minimal differential algebra $(\bigwedge V, d)$ such that there is a $G$-equivariant map

$$
\rho:(\bigwedge V, d) \longrightarrow(A, d)
$$

which is a quasi-isomorphism.

Note that a $G$-minimal model is in particular a minimal model.

Proposition 5.1. Let $(A, d)$ be a one-connected G-differential algebra. Then there exists a $G$-minimal model $(\bigwedge V, d)$ of $(A, d)$.

Proof. The construction of a minimal model in Section 4 works in the context of $G$-differential algebras. All we need is to substitute the vector spaces $V^{n}$ in the construction by $G$ representations. We note that the reductivity of the group $G$ ensures that any short exact sequence of $G$ modules splits.

Let $\left(X, x_{0}\right)$ be a one-pointed compact connected Riemann surface of genus $g \geq 2$, and, as in Section 2 , let $\mathcal{N}_{X}$ be the moduli space of stable vector bundles over $X$ of rank two and fixed determinant $\mathcal{O}_{X}\left(x_{0}\right)$. Then the mapping class group acts on the cohomology $\operatorname{ring} H^{*}\left(\mathcal{N}_{X}, \mathbb{Q}\right)$, with the action factoring through an action of $\operatorname{Sp}(2 g, \mathbb{Z})$; moreover, this action extends to an action of $\operatorname{Sp}(2 g, \mathbb{C})$ on $H^{*}\left(\mathcal{N}_{X}, \mathbb{C}\right)$ (Proposition 2.1). We will now show that a similar result holds for the rational homotopy groups.

Theorem 5.2. The mapping class group acts on the homotopy groups $\pi_{*}\left(\mathcal{N}_{X}\right)$. The induced action on the rational homotopy groups $\pi_{*}\left(\mathcal{N}_{X}\right) \otimes_{\mathbb{Z}} \mathbb{Q}$ factors through an action of the symplectic group $\operatorname{Sp}(2 g, \mathbb{Z})$. This action extends uniquely to an action of $\operatorname{Sp}(2 g, \mathbb{C})$ on $\pi_{*}\left(\mathcal{N}_{X}\right) \otimes_{\mathbb{Z}} \mathbb{C}$. 
Let $(\bigwedge V, d)$ be the $\operatorname{Sp}(2 g, \mathbb{C})$-minimal model, provided by Proposition 5.1 , for the one-connected $\operatorname{Sp}(2 g, \mathbb{C})$-differential algebra $\left(H^{*}\left(\mathcal{N}_{X}, \mathbb{C}\right), 0\right)$. Then

$$
V^{n} \cong\left(\pi_{n}\left(\mathcal{N}_{X}\right) \otimes_{\mathbb{Z}} \mathbb{C}\right)^{*}
$$

as $\operatorname{Sp}(2 g, \mathbb{C})$-modules.

Proof. First note that the formality of $\mathcal{N}_{X}$ (Theorem 4.1) means that $(\bigwedge V, d)$ is also the minimal model of $\mathcal{N}_{X}$.

Now, let $\eta \in \Gamma_{g}^{1}$, where $\Gamma_{g}^{1}$ is the mapping class group of $\left(X, x_{0}\right)$. As we noted prior to Proposition 2.1, the element $\eta$ acts on $\mathcal{N}_{X}$ by a diffeomorphism $f_{\eta}: \mathcal{N}_{X} \longrightarrow \mathcal{N}_{X}$. Hence we have an action on the free homotopy groups of $\mathcal{N}_{X}$. As $\mathcal{N}_{X}$ is simply connected, the free homotopy groups of $\mathcal{N}_{X}$ coincide with the homotopy groups of $\mathcal{N}_{X}$. So we have an induced map

$$
\rho(\eta): \pi_{*}\left(\mathcal{N}_{X}\right) \longrightarrow \pi_{*}\left(\mathcal{N}_{X}\right)
$$

The diffeomorphism $f_{\eta}$ induces a map on differential forms,

$$
f_{\eta}^{*}:\left(\Omega^{*}\left(\mathcal{N}_{X}, \mathbb{C}\right), d\right) \longrightarrow\left(\Omega^{*}\left(\mathcal{N}_{X}, \mathbb{C}\right), d\right)
$$

which lifts to a map on the minimal model

$$
\widehat{f}_{\eta}^{*}:(\bigwedge V, d) \longrightarrow(\bigwedge V, d)
$$

Such a lift is not unique; it is only unique up to homotopy of maps of differential algebras [5]. However, the induced map on the indecomposables,

$$
\tilde{f}_{\eta}^{*}: V=\frac{\bigwedge V}{\bigwedge^{\geq 2} V} \longrightarrow V=\frac{\bigwedge V}{\bigwedge^{\geq 2} V}
$$

is unique [15, Proposition 2.12], and moreover, it coincides with the dual of the map

$$
\rho(\eta) \otimes \mathbb{C}: \pi_{*}\left(\mathcal{N}_{X}\right) \otimes_{\mathbb{Z}} \mathbb{C} \longrightarrow \pi_{*}\left(\mathcal{N}_{X}\right) \otimes_{\mathbb{Z}} \mathbb{C}
$$

under the isomorphism of vector spaces $V^{n} \cong\left(\pi_{n}\left(\mathcal{N}_{X}\right) \otimes_{\mathbb{Z}} \mathbb{C}\right)^{*}($ see $[5$, p. 259]). 
Let

$$
\pi: \Gamma_{g}^{1} \longrightarrow \operatorname{Aut}\left(H_{1}(X, \mathbb{Z})\right)=\operatorname{Sp}(2 g, \mathbb{Z})
$$

be the natural projection of the mapping class group onto the symplectic group. Then the automorphism of cohomology

$$
\bar{f}_{\eta}^{*}: H^{*}\left(\mathcal{N}_{X}, \mathbb{C}\right) \longrightarrow H^{*}\left(\mathcal{N}_{X}, \mathbb{C}\right)
$$

induced by $f_{\eta}^{*}$ in Equation (5.1) coincides with the action of $\pi(\eta)$ on the cohomology, where $\pi$ is the earlier projection. The map $\widehat{f}_{\eta}^{*}$ in equation (5.2) evidently induces the previous automorphism $\bar{f}_{\eta}^{*}$.

The minimal model $(\bigwedge V, d)$ has an action of $\operatorname{Sp}(2 g, \mathbb{Z})$. Indeed, by Proposition 5.1, the group $\operatorname{Sp}(2 g, \mathbb{C})$ acts on $(\bigwedge V, d)$ and this restricts to an action of $\operatorname{Sp}(2 g, \mathbb{Z}) \subset \operatorname{Sp}(2 g, \mathbb{C})$. The homomorphism $\bar{f}_{\eta}^{*}$ is induced by the action of $\pi(\eta)$ on $(\bigwedge V, d)$, where $\pi$ is the projection in Equation (5.4). Therefore the map

$$
\tilde{f}_{\eta}^{*}: V^{n} \longrightarrow V^{n}
$$

defined in Equation (5.3) coincides with the action of $\pi(\eta)$ on $V^{n}$. Hence, under the isomorphism $V^{n} \cong\left(\pi_{n}\left(\mathcal{N}_{X}\right) \otimes_{\mathbb{Z}} \mathbb{C}\right)^{*}$, the actions of $\pi(\eta)$ and $(\rho(\eta) \otimes \mathbb{C})^{*}$ coincide.

If $\eta \in \Gamma_{g}^{1}$ belongs to the Torelli group, then $\rho(\eta) \otimes \mathbb{C}$ must be the identity map of $\pi_{n}\left(\mathcal{N}_{X}\right) \otimes_{\mathbb{Z}} \mathbb{C}$, and hence $\rho(\eta) \otimes \mathbb{Q}=\operatorname{Id}$ on $\pi_{n}\left(\mathcal{N}_{X}\right) \otimes_{\mathbb{Z}} \mathbb{Q}$. This proves that the action of the mapping class group on $\pi_{*}\left(\mathcal{N}_{X}\right) \otimes_{\mathbb{Z}} \mathbb{Q}$ factors through an action of $\operatorname{Sp}(2 g, \mathbb{Z})$. Moreover, this action coincides with the restriction of the $\operatorname{Sp}(2 g, \mathbb{C})$ action on $V^{n}$ to the subgroup $\operatorname{Sp}(2 g, \mathbb{Z}) \subset$ $\operatorname{Sp}(2 g, \mathbb{C})$ under the isomorphism $\left(\pi_{n}\left(\mathcal{N}_{X}\right) \otimes_{\mathbb{Z}} \mathbb{C}\right)^{*} \cong V^{n}$. So the action of $\operatorname{Sp}(2 g, \mathbb{Z})$ on $\pi_{*}\left(\mathcal{N}_{X}\right) \otimes_{\mathbb{Z}} \mathbb{C}$ extends to an action of $\operatorname{Sp}(2 g, \mathbb{C})$. Since $\operatorname{Sp}(2 g, \mathbb{Z})$ is Zariski dense in $\operatorname{Sp}(2 g, \mathbb{C})[3]$, the extension is unique. This completes the proof of the theorem.

Let $G=\operatorname{Sp}(2 g, \mathbb{C})$, and let $(\bigwedge V, d)$ be the $G$-minimal model of $\left(H^{*}\left(\mathcal{N}_{X}\right.\right.$, $\mathbb{C}), 0)$. Then we may decompose $V^{n}$ into irreducible $G$ representations. Let $\left\{\Gamma_{i}\right\}_{i \in \Lambda}$ be a complete set of irreducible $G$ representations, where $\Lambda$ parametrizes the isomorphism classes of irreducible $G$ representations. So

$$
V^{n}=\bigoplus_{i \in \Lambda} a_{i, n} \Gamma_{i}
$$

for some set of integers $a_{i, n} \geq 0$. 


\section{The minimal model of $\mathcal{N}_{X}$ for $g=2$}

In this section we will assume that $X$ is a compact connected Riemann surface of genus two. In this case, the moduli space $\mathcal{N}_{X}$, whose dimension is now three, can be described explicitly $[17,13]$. It turns out to be isomorphic to the intersection of two quadrics in $\mathbb{P}^{5}$.

The integral cohomology ring of $\mathcal{N}_{X}$ has no torsion [16, Section 10]. Let $h \in H^{2}\left(\mathcal{N}_{X}, \mathbb{Z}\right)$ be the hyperplane class. Note that, by the Lefschetz hyperplane theorem, $H^{2}\left(\mathcal{N}_{X}, \mathbb{Z}\right)=H^{2}\left(\mathbb{P}^{5}, \mathbb{Z}\right)=\mathbb{Z}$. So $h=\alpha$, the generator of the ample cone. The intersection of two quadrics in $\mathbb{P}^{5}$ contains many lines $\mathbb{P}^{1} \subset \mathcal{N}_{X} \subset \mathbb{P}^{5}$. Let $l \in H^{4}\left(\mathcal{N}_{X}, \mathbb{Z}\right)$ be the Poincaré dual of such a line. Then $h \cup l=\left[\mathcal{N}_{X}\right]$, so $H^{4}\left(\mathcal{N}_{X}, \mathbb{Z}\right) \cong \mathbb{Z}$ is generated by $l$. We have $h^{3}=$ $h \cup h \cup h=4\left[\mathcal{N}_{X}\right]$, as the degree of $\mathcal{N}_{X} \subset \mathbb{P}^{5}$ is 4 . Therefore, we conclude that $h \cup h=4 l$. Finally, $H^{3}\left(\mathcal{N}_{X}, \mathbb{Z}\right) \cong H^{1}(X, \mathbb{Z})^{*}$, so $H^{3}\left(\mathcal{N}_{X}, \mathbb{Z}\right) \cong W_{0}$, the standard $\operatorname{Sp}(4, \mathbb{Z})$ representation $W_{0}=\mathbb{Z}^{4}$. Moreover, the pairing

$$
H^{3}\left(\mathcal{N}_{X}, \mathbb{Z}\right) \otimes_{\mathbb{Z}} H^{3}\left(\mathcal{N}_{X}, \mathbb{Z}\right) \longrightarrow H^{6}\left(\mathcal{N}_{X}, \mathbb{Z}\right) \cong \mathbb{Z}
$$

is perfect (Poincaré duality) and $\operatorname{Sp}(4, \mathbb{Z})$-equivariant, so it is equivalent to the standard symplectic form on $W_{0}$. The conclusion is that

$$
\begin{aligned}
& H^{0}\left(\mathcal{N}_{X}, \mathbb{Z}\right)=\langle 1\rangle, \\
& H^{1}\left(\mathcal{N}_{X}, \mathbb{Z}\right)=0, \\
& H^{2}\left(\mathcal{N}_{X}, \mathbb{Z}\right)=\langle h\rangle, \\
& H^{3}\left(\mathcal{N}_{X}, \mathbb{Z}\right) \cong W_{0}, \\
& H^{4}\left(\mathcal{N}_{X}, \mathbb{Z}\right)=\langle l\rangle, \\
& H^{5}\left(\mathcal{N}_{X}, \mathbb{Z}\right)=0, \\
& H^{6}\left(\mathcal{N}_{X}, \mathbb{Z}\right)=\left\langle\left[\mathcal{N}_{X}\right]\right\rangle .
\end{aligned}
$$

This can also be seen by using Proposition 3.1, at least for rational coefficients. Since $I_{1}=(\alpha, \beta, \gamma)$ and $I_{2}=\left(\alpha^{2}+\beta, \alpha \beta+\gamma, \alpha \gamma\right)$, Proposition 3.1 says that

$$
H^{*}\left(\mathcal{N}_{X}, \mathbb{Q}\right)=\frac{\mathbb{Q}[\alpha, \beta, \gamma]}{I_{2}} \oplus\left(W \otimes \frac{\mathbb{Q}[\alpha, \beta, \gamma]}{I_{1}}\right) \cong \frac{\mathbb{Q}[\alpha]}{\left(\alpha^{4}\right)} \oplus W
$$

where $\beta=-\alpha^{2}$ and $\gamma=\alpha^{3}$ in this ring, and $\gamma_{i} \cup \gamma_{j}=-\frac{1}{4}\left(\gamma_{i} \cdot \gamma_{j}\right) \alpha^{3}$, for any $\gamma_{i}, \gamma_{j} \in W$. Note that $\beta=-4 l$. Note that $W=W_{0} \otimes_{\mathbb{Z}} \mathbb{Q}$ is the standard $\mathbb{Q}$ representation of $\operatorname{Sp}(4, \mathbb{Z})$. 
Now we pass on to compute the minimal model $(\bigwedge V, d)$ of $\mathcal{N}_{X}$ by computing the minimal model of its cohomology algebra $H^{*}\left(\mathcal{N}_{X}, \mathbb{C}\right)$. This is possible because $\mathcal{N}_{X}$ is formal by Theorem 4.1. By Proposition $5.1,(\bigwedge V, d)$ is a $G$ minimal model for $G=\operatorname{Sp}(4, \mathbb{C})$.

The irreducible representations of $\operatorname{Sp}(4, \mathbb{C})$ are labeled by pairs $(a, b)$, $a, b \geq 0$, such that the corresponding representation $\Gamma_{a, b}$ has highest weight $a L_{1}+b\left(L_{1}+L_{2}\right)=(a+b) L_{1}+b L_{2}$, where $L_{1}$ and $L_{2}$ are the orthogonal generators (with respect to the Killing form) of the weight lattice; see [7, Part III, Section 16].

The standard representation $W_{c}=W \otimes_{\mathbb{Q}} \mathbb{C}=\mathbb{C}^{4}$ of $\operatorname{Sp}(4, \mathbb{C})$ is $W_{c}=$ $\Gamma_{1,0}$, whereas the irreducible $\operatorname{Sp}(4, \mathbb{C})$ representation $\wedge_{0}^{2} W_{c}$ is $\Gamma_{0,1}$. Some easy cases are dealt with in [7, Part III, Section 16],

$$
\begin{aligned}
& \wedge^{2} \Gamma_{1,0}=\wedge_{0}^{2} W_{c} \oplus \mathbb{C}=\Gamma_{0,1} \oplus \Gamma_{0,0}, \\
& \operatorname{Sym}^{a} \Gamma_{1,0}=\Gamma_{a, 0}, \\
& \Gamma_{0,1} \otimes \Gamma_{1,0}=W_{c} \otimes \wedge_{0}^{2} W_{c}=\Gamma_{1,1} \oplus \Gamma_{1,0} .
\end{aligned}
$$

We define a partial order in the set of weights of $\operatorname{Sp}(4, \mathbb{C})$ as follows:

$$
(a, b) \leq(c, d) \Longleftrightarrow\left\{\begin{array}{l}
a+b \leq c+d, \\
a+2 b \leq c+2 d .
\end{array}\right.
$$

This corresponds to the fact that the weights of the representation $\Gamma_{a, b}$ are a subset of the convex hull of the weights of $\Gamma_{c, d}$. Otherwise said, $(a, b) \geq 0$ means that the highest weight $(a+b) L_{1}+b L_{2}$ is a linear combination with non-negative coefficients of the positive roots (see [7]). (We point out that this order is defined in [10, p. 47] with the difference that in $[10],(a, b) \geq 0$ means that $(a+b) L_{1}+b L_{2}$ is a linear combination with non-negative integer coefficients of the positive roots. This is equivalent to $a+b \geq 0, a+2 b \geq 0$ and $a+2 b \equiv 0(\bmod 2)$.)

In particular, for representations $\Gamma_{a_{1}, b_{1}}$ and $\Gamma_{a_{2}, b_{2}}$, the sub-representations $\Gamma_{c, d}$ of the tensor product $\Gamma_{a_{1}, b_{1}} \otimes \Gamma_{a_{2}, b_{2}}$ satisfy the condition

$$
(c, d) \leq\left(a_{1}, b_{1}\right)+\left(a_{2}, b_{2}\right)=\left(a_{1}+a_{2}, b_{1}+b_{2}\right),
$$

and furthermore, there is exactly one sub-representation (the Cartan component) satisfying the equality. Note that this says in particular that $\Gamma_{a, b} \subset$ $W_{c}^{\otimes a} \otimes\left(\wedge_{0}^{2} W_{c}\right)^{\otimes b}$ appears with multiplicity 1 .

We compute the $\operatorname{Sp}(4, \mathbb{C})$-minimal model $(\bigwedge V, d)$ following the mechanism laid out in Section 4 and Proposition 5.1. 
Proposition 6.1. Let $(\bigwedge V, d)$ be the minimal model of $\mathcal{N}_{X}$ for a curve $X$ of genus $g=2$. Then we have, as $\operatorname{Sp}(4, \mathbb{Z})$-representations,

$$
\begin{aligned}
V^{2} & =\Gamma_{0,0} \\
V^{3} & =\Gamma_{1,0} \\
V^{4} & =\Gamma_{1,0} \\
V^{5} & =\Gamma_{0,1} \oplus \Gamma_{0,0} \\
V^{6} & =\Gamma_{2,0} \oplus \Gamma_{0,1} \\
V^{7} & =\Gamma_{1,1} \oplus \Gamma_{2,0} \oplus \Gamma_{1,0} .
\end{aligned}
$$

Proof. Abbreviating $H^{*}\left(\mathcal{N}_{X}\right)$ for $H^{*}\left(\mathcal{N}_{X}, \mathbb{C}\right)$, we have

$$
V^{2}=C^{2}=H^{2}\left(\mathcal{N}_{X}\right)=\langle h\rangle \cong \Gamma_{0,0} .
$$

Recall that $\left.d\right|_{C^{n}}=0$, for any $n$.

In the next step, we have $V^{3}=C^{3} \oplus N^{3}$, with

$$
\begin{aligned}
& C^{3}=\operatorname{coker}\left(H^{3}\left(\bigwedge V^{\leq 2}\right)=0 \longrightarrow H^{3}\left(\mathcal{N}_{X}\right)\right)=H^{3}\left(\mathcal{N}_{X}\right) \cong W_{c} \cong \Gamma_{1,0}, \\
& N^{3}=\operatorname{ker}\left(H^{4}\left(\bigwedge V^{\leq 2}\right)=\left\langle h^{2}\right\rangle \longrightarrow H^{4}\left(\mathcal{N}_{X}\right)=\left\langle h^{2}\right\rangle\right)=0 .
\end{aligned}
$$

For $n=4$, we have $V^{4}=C^{4} \oplus N^{4}$, with

$$
\begin{aligned}
C^{4} & =\operatorname{coker}\left(H^{4}\left(\bigwedge V^{\leq 3}\right)=\left\langle h^{2}\right\rangle \longrightarrow H^{4}\left(\mathcal{N}_{X}\right)\left\langle h^{2}\right\rangle\right)=0 \\
N^{4} & =\operatorname{ker}\left(H^{5}\left(\bigwedge V^{\leq 3}\right) \longrightarrow H^{5}\left(\mathcal{N}_{X}\right)=0\right)=H^{5}\left(\bigwedge V^{\leq 3}\right) \\
& =V^{3} \cdot V^{2} \cong \Gamma_{1,0} \otimes \Gamma_{0,0}=\Gamma_{1,0} .
\end{aligned}
$$

The differential $d: N^{4} \longrightarrow V^{3} \cdot V^{2} \subset \bigwedge V$ is an isomorphism.

We continue with $V^{5}=C^{5} \oplus N^{5}$, where

$$
\begin{aligned}
C^{5} & =\operatorname{coker}\left(H^{5}\left(\bigwedge V^{\leq 4}\right) \longrightarrow H^{5}\left(\mathcal{N}_{X}\right)=0\right)=0 \\
N^{5} & =\operatorname{ker}\left(H^{6}\left(\bigwedge V^{\leq 4}\right)=\wedge^{2} V^{3} \oplus\left\langle h^{3}\right\rangle \longrightarrow H^{6}\left(\mathcal{N}_{X}\right)=\left\langle h^{3}\right\rangle\right) \\
& \cong \wedge^{2} V^{3} \cong \wedge^{2} \Gamma_{1,0}=\Gamma_{0,1} \oplus \Gamma_{0,0}
\end{aligned}
$$

The differential $d: N^{5} \longrightarrow \wedge^{2} V^{3} \oplus\left\langle h^{3}\right\rangle$ is an isomorphism of $N^{5}$ with the kernel of the map $\wedge^{2} V^{3} \oplus\left\langle h^{3}\right\rangle \longrightarrow\left\langle h^{3}\right\rangle$. This map is the sum of a multiple of the intersection product $\wedge^{2} V^{3} \longrightarrow \mathbb{C} \cong\left\langle h^{3}\right\rangle$ in the first summand, and the identity in the second summand. 
For $n=6$, we have $V^{6}=C^{6} \oplus N^{6}$. Now

$$
C^{6}=\operatorname{coker}\left(H^{6}\left(\bigwedge V^{\leq 5}\right) \rightarrow H^{6}\left(\mathcal{N}_{X}\right)=\left\langle h^{3}\right\rangle\right)=0,
$$

since $h^{3} \in H^{6}\left(\bigwedge V^{\leq 5}\right)$. Moreover $C^{k}=0$ for $k>6$ since $H^{k}\left(\mathcal{N}_{X}\right)=0$. Also for all $k \geq 6$, we have $N^{k}=H^{k+1}\left(\bigwedge V^{\leq(k-1)}\right)$, since $H^{k+1}\left(\mathcal{N}_{X}\right)=0$. Now

$$
\begin{aligned}
& \left(\bigwedge V^{\leq 5}\right)^{6}=\left(V^{3} \cdot V^{3}\right) \oplus\left(V^{2} \cdot V^{2} \cdot V^{2}\right) \oplus\left(V^{4} \cdot V^{2}\right), \\
& \left(\bigwedge V^{\leq 5}\right)^{7}=\left(V^{4} \cdot V^{3}\right) \oplus\left(V^{3} \cdot V^{2} \cdot V^{2}\right) \oplus\left(V^{5} \cdot V^{2}\right) .
\end{aligned}
$$

The space of coboundaries is $B^{7}\left(\bigwedge V^{\leq 5}\right)=d\left(V^{4} \cdot V^{2}\right)=V^{3} \cdot V^{2} \cdot V^{2}$. The differential $d$ maps $\left(V^{4} \cdot V^{3}\right) \oplus\left(V^{5} \cdot V^{2}\right)$ onto $\wedge^{2} V^{3} \cdot V^{2} \oplus\left\langle h^{4}\right\rangle$, and it has kernel isomorphic to $\operatorname{ker}\left(V^{4} \cdot V^{3} \longrightarrow\left\langle h^{4}\right\rangle\right)$. But

$$
V^{4} \cdot V^{3}=V^{4} \otimes V^{3} \cong \Gamma_{1,0} \otimes \Gamma_{1,0}=\operatorname{Sym}^{2} \Gamma_{1,0} \oplus \wedge^{2} \Gamma_{1,0} \cong \Gamma_{2,0} \oplus \Gamma_{0,1} \oplus \Gamma_{0,0}
$$

So the conclusion is that

$$
N^{6}=H^{7}\left(\bigwedge V^{\leq 5}\right)=\frac{Z^{7}\left(\bigwedge V^{\leq 5}\right)}{B^{7}\left(\bigwedge V^{\leq 5}\right)} \cong \Gamma_{2,0} \oplus \Gamma_{0,1},
$$

and the differential $d: N^{6} \longrightarrow\left(V^{4} \cdot V^{3}\right) \oplus\left(V^{5} \cdot V^{2}\right)$ is the sum of the two maps $d: N^{6}=\Gamma_{2,0} \oplus \Gamma_{0,1} \longrightarrow V^{4} \cdot V^{3}=\Gamma_{2,0} \oplus \Gamma_{0,1} \oplus \Gamma_{0,0}$ which is injective, and $d: N^{6}=\Gamma_{2,0} \oplus \Gamma_{0,1} \longrightarrow V^{5} \cdot V^{2}=\Gamma_{0,1} \oplus \Gamma_{0,0}$ mapping onto the $\Gamma_{0,1}$ summand.

The next case is $V^{7}=C^{7} \oplus N^{7}=N^{7}$. Then

$$
\begin{aligned}
\left(\bigwedge V^{\leq 6}\right)^{7}= & \left(V^{5} \cdot V^{2}\right) \oplus\left(V^{4} \cdot V^{3}\right) \oplus\left(V^{3} \cdot V^{2} \cdot V^{2}\right) \\
\left(\bigwedge V^{\leq 6}\right)^{8}= & \left(V^{6} \cdot V^{2}\right) \oplus\left(V^{5} \cdot V^{3}\right) \oplus\left(V^{4} \cdot V^{4}\right) \oplus\left(V^{4} \cdot V^{2} \cdot V^{2}\right) \\
& \oplus\left(V^{3} \cdot V^{3} \cdot V^{2}\right) \oplus\left\langle h^{4}\right\rangle .
\end{aligned}
$$

The space of coboundaries is $B^{8}\left(\bigwedge V^{\leq 6}\right)=\left(V^{3} \cdot V^{3} \cdot V^{2}\right) \oplus\left\langle h^{4}\right\rangle$, from our knowledge of $d$ on both $V^{5}$ and $V^{4}$. To compute

$$
\begin{aligned}
N^{7} & =H^{8}\left(\bigwedge V^{\leq 7}\right)=\frac{Z^{8}\left(\bigwedge V^{\leq 6}\right)}{B^{8}\left(\bigwedge V^{\leq 6}\right)}= \\
& =\operatorname{ker}\left(d:\left(V^{6} \cdot V^{2}\right) \oplus\left(V^{5} \cdot V^{3}\right) \oplus\left(V^{4} \cdot V^{4}\right) \oplus\left(V^{4} \cdot V^{2} \cdot V^{2}\right) \longrightarrow \bigwedge V\right),
\end{aligned}
$$


we look at each summand,

$$
\begin{aligned}
& d: V^{5} \cdot V^{3} \longrightarrow \wedge^{3} V^{3} \oplus V^{3} \cdot\left(V^{2}\right)^{3}, \\
& d: V^{4} \cdot V^{2} \cdot V^{2} \stackrel{\simeq}{\longrightarrow} V^{3} \cdot\left(V^{2}\right)^{3}, \\
& d: V^{6} \cdot V^{2} \hookrightarrow\left(V^{4} \cdot V^{3} \cdot V^{2}\right) \oplus\left(V^{5} \cdot V^{2} \cdot V^{2}\right) \\
& d: V^{4} \cdot V^{4} \hookrightarrow V^{4} \cdot V^{3} \cdot V^{2} .
\end{aligned}
$$

So $N^{7}=K_{1} \oplus K_{2}$, where $K_{1}=\operatorname{ker}\left(\left(V^{5} \cdot V^{3}\right) \oplus\left(V^{4} \cdot V^{2} \cdot V^{2}\right) \longrightarrow \bigwedge V\right)$ and $K_{2}=\operatorname{ker}\left(\left(V^{6} \cdot V^{2}\right) \oplus\left(V^{4} \cdot V^{4}\right) \longrightarrow \bigwedge V\right)$. Clearly, $K_{1} \cong \operatorname{ker}\left(V^{5} \otimes V^{3} \longrightarrow\right.$ $\left.\wedge^{3} V^{3}\right)$, but $V^{5} \otimes V^{3} \cong\left(\Gamma_{0,1} \oplus \Gamma_{0,0}\right) \otimes \Gamma_{1,0}=\Gamma_{1,1} \oplus \Gamma_{1,0} \oplus \Gamma_{1,0}$ and $\wedge^{3} V^{3} \cong$ $V^{3} \cong \Gamma_{1,0}$, so $K_{1} \cong \Gamma_{1,1} \oplus \Gamma_{1,0}$. On the other hand, $d$ maps $V^{4} \cdot V^{4}=$ Sym $^{2} V^{4} \cong \Gamma_{2,0}$ to the corresponding summand in $V^{4} \cdot V^{3} \cdot V^{2} \cong \Gamma_{1,0} \otimes \Gamma_{1,0}=$ $\Gamma_{2,0} \oplus \Gamma_{0,1} \oplus \Gamma_{0,0}$, and $d$ maps $V^{6} \cdot V^{2} \cong \Gamma_{2,0} \oplus \Gamma_{0,1}$ injectively to $\left(V^{4}\right.$. $\left.V^{3} \cdot V^{2}\right) \oplus\left(V^{5} \cdot V^{2} \cdot V^{2}\right) \cong\left(\Gamma_{2,0} \oplus \Gamma_{0,1} \oplus \Gamma_{0,0}\right) \oplus\left(\Gamma_{0,1} \oplus \Gamma_{0,0}\right)$. Thus $K_{2} \cong$ $\Gamma_{2,0}$. This concludes that $N^{7}=\Gamma_{1,1} \oplus \Gamma_{2,0} \oplus \Gamma_{1,0}$, and the proof of the proposition is complete.

We may carry on the process as long as we want, but the calculations get more involved, since we must keep track of the irreducible summands of $\bigwedge V$ onto which $\left.d\right|_{V_{n}}$ maps for each $n$. It is easier to find the "leading representation". We need a preliminary result.

Lemma 6.2. For any $\operatorname{Sp}(4, \mathbb{C})$-irreducible representation $\Gamma_{a, b}, a, b \geq 0$, if $\Gamma_{a, b} \subset V^{n}$, then $n \geq n(a, b)$, where

$$
n(a, b)= \begin{cases}2 a+4 b+1, & \text { if } b \geq 1 \text { or }(a, b)=(1,0), \\ 2 a+2, & \text { if } b=0 \text { and } a \neq 1 .\end{cases}
$$

Proof. We shall prove this by induction on $n$. By Proposition 6.1, the result is true for $1 \leq n \leq 7$. So suppose $n \geq 8$. Let $U \subset V^{n}$ be a sub-representation with $U \cong \Gamma_{a, b}$. We want to prove that $n(a, b) \leq n$, so we may assume that $(a, b) \neq(0,0),(1,0),(0,1),(1,1)$ and $(2,0)$. As $H^{n}\left(\mathcal{N}_{X}\right)=0$, we have that $C^{n}=0$. So $V^{n}=N^{n}$, and in particular $d: V^{n} \longrightarrow(\bigwedge V)^{n+1}$ is injective. Hence

$$
U \cong d(U) \subset d\left(V^{n}\right) \subset\left(\bigwedge^{\geq 2} V\right)^{n+1}=\sum_{n_{1}+\ldots+n_{r}=n+1, r \geq 2} V^{n_{1}} \cdots V^{n_{r}} .
$$

The projection of $d(U)$ to some of these summands must be non-zero. Hence there exists

$$
U^{\prime} \subset V^{n_{1}} \cdots V^{n_{r}} \subset V^{n_{1}} \otimes \cdots \otimes V^{n_{r}}
$$


for some $r \geq 2$, with $n_{1}+\cdots+n_{r}=n+1, U^{\prime} \cong U \cong \Gamma_{a, b}$. Note that all $n_{i}<n$, because $n_{i} \geq 2,1 \leq i \leq r$. Decomposing each $V^{n_{i}}$ into $\operatorname{Sp}(4, \mathbb{C})$ irreducible representations, there must be $\left(a_{1}, b_{1}\right), \ldots,\left(a_{r}, b_{r}\right)$ such that

$$
\Gamma_{a, b} \subset \Gamma_{a_{1}, b_{1}} \otimes \cdots \otimes \Gamma_{a_{r}, b_{r}}
$$

with $\Gamma_{a_{i}, b_{i}} \subset V^{n_{i}}$. Applying the induction hypothesis it follows that $n_{i} \geq$ $n\left(a_{i}, b_{i}\right)$ for all $1 \leq i \leq r$. We note that Equation (6.1) implies that $(a, b) \leq$ $\left(a_{1}, b_{1}\right)+\cdots+\left(a_{r}, b_{r}\right)$, that is,

$$
\left\{\begin{array}{l}
a+b \leq \sum\left(a_{i}+b_{i}\right) \\
a+2 b \leq \sum\left(a_{i}+2 b_{i}\right)
\end{array}\right.
$$

If $a, b \geq 1$, we have

$$
\begin{aligned}
n+1 & =n_{1}+\cdots+n_{r} \\
& \geq n\left(a_{1}, b_{1}\right)+\cdots+n\left(a_{r}, b_{r}\right) \\
& \geq \sum_{i=1}^{r}\left(2 a_{i}+4 b_{i}+1\right) \\
& \geq 2 a+4 b+r \\
& \geq 2 a+4 b+2 \\
& =n(a, b)+1,
\end{aligned}
$$

using Equation (6.2). So $n \geq n(a, b)$ in this case.

If $b=0, a \geq 3$, then Equation (6.3) proves that $n+1 \geq n(a, b)$. If there is equality, then $r=2, n_{i}=n\left(a_{i}, b_{i}\right)=2 a_{i}+4 b_{i}+1$ and $a=a+2 b=$ $\sum\left(a_{i}+2 b_{i}\right)$, for all $i$. Since $a=a+b \leq \sum\left(a_{i}+b_{i}\right)$, we get $\sum\left(a_{i}+2 b_{i}\right)=$ $a \leq \sum\left(a_{i}+b_{i}\right)$, so $b_{i}=0$ for all $i$. As also $n_{i}=n\left(a_{i}, 0\right)=2 a_{i}+1$, we have that $a_{i}=1, n_{i}=3$. But then $a=2$ which is a case treated before.

Theorem 6.3. Let $n \geq 4$. The decomposition of $V^{n}$ into a direct sum of irreducible $\operatorname{Sp}(4, \mathbb{C})$-representations is as follows:

(i) If $n=2 m$ is even, then

$$
V^{n}=\Gamma_{m-1,0} \oplus\left(\bigoplus_{(a, b)<(m-1,0)} n_{a b} \Gamma_{a, b}\right)
$$

with $n_{a b} \geq 0$. 
(ii) If $n=2 m+1$ is odd, then

$$
V^{n}=\Gamma_{m-2,1} \oplus\left(\bigoplus_{(a, b)<(m-2,1)} n_{a b} \Gamma_{a, b}\right)
$$

with $n_{a b} \geq 0$.

Proof. (i) Let $n=2 m \geq 4$. By Lemma 6.2 , if $\Gamma_{a, b} \subset V^{n}$ then $2 a+4 b+1 \leq$ $n=2 m$. This implies that

$$
(a, b) \leq(m-1,0)
$$

since $a+2 b \leq m-1$ and $a+b \leq a+2 b \leq m-1$. So the leading representation in $V^{n}$ is $\Gamma_{m-1,0}$. We will show that it actually appears and that its multiplicity is one.

To see that there is $\Gamma_{m-1,0} \subset V^{n}$, we shall prove by induction on $m$ that there is a sub-representation $U_{m-1} \subset V^{n}, U_{m-1} \cong \Gamma_{m-1,0}$, such that $d\left(U_{m-1}\right) \subset V^{n-2} \cdot V^{3}$. By Proposition 6.1, this is true for $m=2,3$. Assume that it is true for $m-1 \geq 3$ and let us prove it for $m$. So $U_{m-2} \subset V^{2 m-2}$ and $d\left(U_{m-2}\right) \subset V^{2 m-4} \cdot V^{3}$. Then $d: U_{m-2} \cdot V^{3} \longrightarrow V^{2 m-4} \cdot V^{3} \cdot V^{3} \subset \bigwedge V$. But

$$
U_{m-2} \cdot V^{3} \cong \Gamma_{m-2,0} \otimes \Gamma_{1,0}
$$

contains a sub-representation $\widetilde{U}_{m-1} \subset U_{m-2} \cdot V^{3}$ such that $\widetilde{U}_{m-1} \cong \Gamma_{m-1,0}$. On the other hand,

$$
V^{2 m-4} \cdot V^{3} \cdot V^{3}=V^{2 m-4} \otimes \wedge^{2} V^{3} \cong V^{2 m-4} \otimes \Gamma_{0,1} \cdot
$$

Decomposing $V^{2 m-4}$ into irreducible representations $\Gamma_{c, d}$, and noting that $(c, d) \leq(m-3,0)$ by induction hypothesis, we see that if $\Gamma_{a, b} \subset V^{2 m-4}$. $V^{3} \cdot V^{3}$ then $\Gamma_{a, b} \subset \Gamma_{c, d} \otimes \Gamma_{0,1}$ for some $(c, d) \leq(m-3,0)$. Thus

$$
(a, b) \leq(c, d)+(0,1)=(c, d+1) \leq(m-3,1)<(m-1,0) .
$$

As a consequence, $\Gamma_{m-1,0} \not \subset V^{2 m-4} \cdot V^{3} \cdot V^{3}$, and so $d\left(\widetilde{U}_{m-1}\right)=0$. This implies that $\widetilde{U}_{m-1} \subset Z^{n+1}(\bigwedge V, d)=B^{n+1}(\bigwedge V, d)$, since $H^{n+1}(\bigwedge V, d)=0$. There must exist a sub-representation $U_{m-1} \subset(\bigwedge V)^{n}$ with $d\left(U_{m-1}\right)=\widetilde{U}_{m-1}$. As $d \operatorname{maps}\left(\bigwedge^{\geq i} V\right)^{n} \longrightarrow\left(\bigwedge^{\geq(i+1)} V\right)^{n+1}$, it cannot be $U_{m-1} \subset\left(\bigwedge^{2} V\right)^{n}$, so the projection of $U_{m-1}$ by $p:(\wedge V)^{n} \longrightarrow V^{n}$ is a sub-representation isomorphic to $\Gamma_{m-1,0}$. (Here we are allowed to substitute $V^{n}$ by $U_{m-1} \oplus p\left(U_{m-1}\right)^{\perp}$, where $p\left(U_{m-1}\right)^{\perp}$ is a $\operatorname{Sp}(4, \mathbb{C})$-invariant complement of $p\left(U_{m-1}\right) \subset V^{n}$; this 
yields an isomorphic minimal model and ensures that $d\left(U_{m-1}\right) \subset$ $\left.V^{2 n-2} \cdot V^{3}\right)$.

Now let us compute the multiplicity of $\Gamma_{m-1,0}$ in $V^{n}$. The argument of the proof of Lemma 6.2 implies that the multiplicity of $\Gamma_{m-1,0}$ in $V^{n}$ is at most the sum of the multiplicities of $\Gamma_{m-1,0}$ in $V^{n_{1}} \cdots V^{n_{r}}$, for the different possibilities $n_{1}+\cdots+n_{r}=n+1, r \geq 2$. Let $(a, b)=(m-1,0)$. As in the proof of Lemma 6.2, for any sub-representation $\Gamma_{m-1,0}$ there are $\left(a_{1}, b_{1}\right), \ldots,\left(a_{r}, b_{r}\right)$ such that

$$
\begin{aligned}
2 m+1 & =n+1=n_{1}+\cdots+n_{r} \\
& \geq n\left(a_{1}, b_{1}\right)+\cdots+n\left(a_{r}, b_{r}\right) \\
& \geq \sum_{i=1}^{r}\left(2 a_{i}+4 b_{i}+1\right) \\
& \geq 2 a+4 b+r=2 m-2+r .
\end{aligned}
$$

In particular $r \leq 3$. If $r=3$, then $n_{i}=n\left(a_{i}, b_{i}\right)=2 a_{i}+4 b_{i}+1$ for all $i$, and $a=a+2 b=\sum\left(a_{i}+2 b_{i}\right)$. Since $a=a+b \leq \sum\left(a_{i}+b_{i}\right)$, we get $b_{i}=0$ for all $i$. This implies that $a_{i}=1$ and $n_{i}=3$. But then $a=m-1=3$ and

$$
\Gamma_{3,0} \not \subset V^{3} \cdot V^{3} \cdot V^{3}=\wedge^{3} V^{3} \cong \wedge^{3} W_{c} \cong W_{c} \cong \Gamma_{1,0} .
$$

If $r=2$, then $2 a+4 b+1 \geq \sum\left(2 a_{i}+4 b_{i}\right) \geq 2 a+4 b$. So $\sum\left(a_{i}+2 b_{i}\right)=$ $a+2 b=a$. As before, this implies that $b_{i}=0$ for all $i$. At most one of the $a_{i}$ s is bigger than 1 , so we can put $\left(a_{1}, b_{1}\right)=(m-2,0),\left(a_{2}, b_{2}\right)=(1,0)$. This corresponds to the summand $\Gamma_{m-2,0} \otimes \Gamma_{1,0} \subset V^{2 m-4} \cdot V^{3}$. This representation contains $\Gamma_{m-1,0}$ with multiplicity one.

Since we know that the multiplicity of $\Gamma_{m-1,0}$ in $V^{n}$ is non-zero, we conclude that it is exactly one.

(ii) Let $n=2 m+1 \geq 5$. By Proposition 6.1, the result holds for $m=$ 2,3 , so assume that $m \geq 4$.

If $\Gamma_{a, b} \subset V^{n}$, then by Lemma 6.2 , we have that $2 a+4 b+1 \leq n=2 m+$ 1 , so $a+2 b \leq m$. This implies that

$$
(a, b) \leq(m-2,1),
$$

since if $b \geq 1$ then $a+b \leq a+2 b-1 \leq m-1$; and if $b=0$ then Lemma 6.2 says that $2 a+2 \leq n=2 m+1$, so $a+b=a \leq m-1$. So the leading representation in $V^{n}$ is $\Gamma_{m-2,1}$. We will show that it actually appears with multiplicity one.

As in the previous case, one can see using induction on $m$ that there is a sub-representation $U_{m-1} \subset V^{2 m+1}$, with $U_{m-1} \cong \Gamma_{m-2,1}$, such that $d\left(U_{m-1}\right)$ $\subset V^{2 m-1} \cdot V^{3}$. 
To compute the multiplicity of $\Gamma_{m-2,1}$ in $V^{n}$, let us find the multiplicity of $\Gamma_{m-2,1}$ in $V^{n_{1}} \cdots V^{n_{r}}$, for $n_{1}+\cdots+n_{r}=n+1, r \geq 2$. As $n=2 m+1=$ $n(m-2,1)$, there must be equality in Equation $(6.3)$ for $(a, b)=(m-2,1)$, which means that $r=2, n_{i}=n\left(a_{i}, b_{i}\right)=2 a_{i}+4 b_{i}+1$ and $\sum\left(a_{i}+2 b_{i}\right)=$ $a+2 b=m$. Since $m-1=a+b \leq \sum\left(a_{i}+b_{i}\right)$, we have $\sum\left(a_{i}+2 b_{i}\right)=m \leq$ $\sum\left(a_{i}+b_{i}\right)+1$, so $\sum b_{i} \leq 1$. As least one $b_{i}$ is zero, say $b_{2}=0$. Then $a_{2}=1$, $n_{2}=3$. Also $m-1 \leq \sum\left(a_{i}+b_{i}\right) \leq a_{1}+2$ and $m=a_{1}+2 b_{1}+1$, implying that $\left(a_{1}, b\right)=(m-3,1)$ or $(m-1,0)$ and $n_{1}=2 a_{1}+4 b_{1}+1=2 m-1$. By induction hypothesis, $\Gamma_{m-1,0} \not \subset V^{2 m-1}$, so the second case is ruled out. The multiplicity of $\Gamma_{m-2,1}$ in $\Gamma_{m-3,1} \otimes \Gamma_{1,0} \subset V^{2 m-1} \cdot V^{3}$ is 1 . This proves that the multiplicity of $\Gamma_{m-2,1}$ in $V^{n}$ is one.

\section{Sub-representations in the minimal model of $\mathcal{N}_{X}$ for $g>2$}

Suppose now that $X$ is a smooth irreducible projective complex curve of genus $g>2$. The action of $\operatorname{Sp}(2 g, \mathbb{C})$ on the cohomology algebra $H^{*}\left(\mathcal{N}_{X}, \mathbb{C}\right)$ of the moduli space $\mathcal{N}_{X}$ gives an action of $\operatorname{Sp}(2 g, \mathbb{C})$ on the minimal model $(\bigwedge V, d)$ of $\mathcal{N}_{X}$, by Proposition 5.1. By Theorem 5.2, the action of $\operatorname{Sp}(2 g, \mathbb{C})$ on the minimal model $(\bigwedge V, d)$ is compatible with the action of $\operatorname{Sp}(2 g, \mathbb{C})$ on the complex homotopy groups $\pi_{*}\left(\mathcal{N}_{X}\right) \otimes_{\mathbb{Z}} \mathbb{C}$.

The isomorphism classes of irreducible $\operatorname{Sp}(2 g, \mathbb{C})$-representations are labeled by $g$-tuples $\left(a_{1}, \ldots, a_{g}\right) \in\left(\mathbb{Z}_{\geq 0}\right)^{g}$ (see $[7$, Part III, Section 17]). The representation corresponding to $\left(a_{1}, \ldots, a_{g}\right)$ is denoted by

$$
\Gamma_{\left(a_{1}, \ldots, a_{g}\right)}=\Gamma_{a_{1} \mathbf{e}_{1}+\cdots+a_{g} \mathbf{e}_{g}}
$$

where $\mathbf{e}_{i}=(0, \ldots, 1, \ldots, 0)$, with 1 in the $i$ th position and 0 elsewhere. The $\operatorname{Sp}(2 g, \mathbb{C})$-module $\Gamma_{\left(a_{1}, \ldots, a_{g}\right)}$ is characterized by its highest weight $\left(a_{1}+\right.$ $\left.a_{2}+\cdots+a_{g}\right) L_{1}+\left(a_{2}+\cdots+a_{g}\right) L_{2}+\cdots+a_{g} L_{g}$, where $\left\{L_{1}, \ldots, L_{g}\right\}$ is the standard basis for the weight lattice.

Let $W_{c}=\mathbb{C}^{2 g}$ be the standard representation of $\operatorname{Sp}(2 g, \mathbb{C})$. Then $W_{c}=$ $\Gamma_{\mathbf{e}_{1}}$, and $\Gamma_{\mathbf{e}_{k}}=\wedge_{0}^{k} W_{c}$ is the complexification of the representation $\wedge_{0}^{k} W$ introduced in Section 3.

We shall use two well-known facts: (i) the representation $\Gamma_{\left(a_{1}, \ldots, a_{g}\right)} \otimes$ $\Gamma_{\left(b_{1}, \ldots, b_{g}\right)}$ contains $\Gamma_{\left(a_{1}+b_{1}, \ldots, a_{g}+b_{g}\right)}$ (actually this is the highest weight representation appearing with multiplicity one); and (ii) the representation $\Gamma_{(k-2,0, \ldots, 0)} \otimes \Gamma_{(0,1, \ldots, 0)}$ does not contain $\Gamma_{(k, 0, \ldots 0)}$ (this holds because the weight $k L_{1}$ does not appear in the tensor product), and the representation $\Gamma_{(k-2,1,0, \ldots, 0)} \otimes \Gamma_{(0,1,0, \ldots, 0)}$ does not contain $\Gamma_{(k, 1,0, \ldots, 0)}$. 
Theorem 7.1. Let $X$ be a complex smooth projective irreducible curve of genus $g>2$. Let $(\bigwedge V, d)$ be the minimal model of the moduli space $\mathcal{N}_{X}$. Then, as $\operatorname{Sp}(2 g, \mathbb{C})$ representations, we have

$$
\begin{aligned}
& V^{2}=\Gamma_{0}, \\
& V^{3}=\Gamma_{\mathbf{e}_{1}}, \\
& V^{4}=\Gamma_{0}, \\
& V^{n}=0, \\
& V^{2 g-1}=\Gamma_{0}, \\
& V^{2 g}=\Gamma_{\mathbf{e}_{1}}, \\
& V^{2 g+1}=\Gamma_{\mathbf{e}_{2}} \oplus \Gamma_{0} .
\end{aligned}
$$

Moreover, for $n \geq 2 g+2$, we have the following.

(i) If $n=2(g+k-1)$ with $k \geq 2$, then $V^{n}$ contains $\Gamma_{k \mathbf{e}_{1}}$.

(ii) If $n=2(g+k)+1$ with $k \geq 1$, then $V^{n}$ contains $\Gamma_{k \mathbf{e}_{1}+\mathbf{e}_{2}}$.

Proof. Clearly, $V^{2}=C^{2}=\langle\alpha\rangle \cong \Gamma_{0}$,

$$
V^{3}=C^{3}=H^{3}\left(\mathcal{N}_{X}\right)=W_{c} \cong \Gamma_{\mathbf{e}_{1}}
$$

and $V^{4}=C^{4}=\langle\beta\rangle \cong \Gamma_{0}$. Now

$$
\bigwedge^{\leq 4}=\bigwedge\left(\alpha, \gamma_{1}, \ldots, \gamma_{2 g}, \beta\right)=\mathbb{A}_{c}
$$

where $\mathbb{A}_{c}=\mathbb{A} \otimes_{\mathbb{Q}} \mathbb{C}$ is the complexification of the rational vector space defined in Lemma 3.2. So the natural homomorphism $\bigwedge V^{\leq 4} \longrightarrow H^{*}\left(\mathcal{N}_{X}\right)$ is surjective. This implies that $C^{n}=0$ and

$$
V^{n}=N^{n}=\operatorname{ker}\left(H^{n+1}\left(\bigwedge V^{<n}\right) \longrightarrow H^{n+1}\left(\mathcal{N}_{X}\right)\right)
$$

for all $n>4$. Since $F_{c}: \mathbb{A}_{c} \longrightarrow H^{*}\left(\mathcal{N}_{X}\right)$ is the complexification of the map $F$ in Equation (3.3), its kernel, $\operatorname{kernel}\left(F_{c}\right)$, has the lowest degree element $q_{g}^{1}$, which is of degree $2 g$. So $V^{n}=N^{n}=0$ for all $5 \leq n \leq 2 g-2$. For $n=2 g-1$, we have

$$
\begin{aligned}
V^{2 g-1} & =\operatorname{ker}\left(H^{2 g}\left(\bigwedge V^{<(2 g-1)}\right)=\mathbb{A}_{c}^{2 g} \longrightarrow H^{n+1}\left(\mathcal{N}_{X}\right)\right)=\left(\operatorname{kernel}\left(F_{c}\right)\right)^{2 g} \\
& =\left\langle q_{g}^{1}\right\rangle \cong \Gamma_{0}
\end{aligned}
$$

with $d: V^{2 g-1} \stackrel{\simeq}{\longrightarrow}\left\langle q_{g}^{1}\right\rangle \subset \bigwedge V$. 
For $n=2 g$, we have

$$
H^{2 g+1}\left(\bigwedge V^{\leq(2 g-1)}\right)=H^{2 g+1}\left(\bigwedge V^{<(2 g-1)}\right)=\mathbb{A}_{c}^{2 g+1},
$$

since $\left(\bigwedge V^{\leq(2 g-1)}\right)^{2 g+1}=\mathbb{A}_{c}^{2 g+1} \oplus\left(V^{2 g-1} \cdot V^{2}\right)$ and the non-zero elements in $V^{2 g-1} \cdot V^{2}$ are not closed. So

$$
V^{2 g}=\left(\operatorname{kernel}\left(F_{c}\right)\right)^{2 g+1}=q_{g-1}^{1} \cdot W_{c} \cong \Gamma_{\mathbf{e}_{1}}
$$

and $d: V^{2 g} \stackrel{\simeq}{\longrightarrow} q_{g-1}^{1} \cdot V^{3} \subset \bigwedge V$.

For $n=2 g+1$, we have

$$
\begin{aligned}
& \left(\bigwedge V^{\leq 2 g}\right)^{2 g+1}=\mathbb{A}_{c}^{2 g+1} \oplus\left(V^{2 g-1} \cdot V^{2}\right) \\
& \left(\bigwedge V^{\leq 2 g}\right)^{2 g+2}=\mathbb{A}_{c}^{2 g+2} \oplus\left(V^{2 g-1} \cdot V^{3}\right) \oplus\left(V^{2 g} \cdot V^{2}\right),
\end{aligned}
$$

with $d: V^{2 g-1} \cdot V^{3} \stackrel{\simeq}{\longrightarrow} q_{g}^{1} \cdot V^{3}$ and $d: V^{2 g} \cdot V^{2} \stackrel{\simeq}{\longrightarrow} \alpha q_{g-1}^{1} \cdot V^{3}$. But $\alpha q_{g-1}^{1}$ and $q_{g}^{1}$ are linearly independent, so we have

$$
H^{2 g+2}\left(\bigwedge V^{\leq 2 g}\right)=\frac{Z^{2 g+2}\left(\bigwedge V^{\leq 2 g}\right)}{B^{2 g+2}\left(\bigwedge V^{\leq 2 g}\right)}=\frac{\mathbb{A}_{c}^{2 g+2}}{\left\langle\alpha q_{g}^{1}\right\rangle}
$$

This gives

$$
V^{2 g+1}=\operatorname{ker}\left(\frac{\mathbb{A}_{c}^{2 g+2}}{\left\langle\alpha q_{g}^{1}\right\rangle} \longrightarrow H^{2 g+2}\left(\mathcal{N}_{X}\right)\right)=\left\langle q_{g}^{2}\right\rangle \oplus q_{g-2}^{1} \cdot \wedge_{0}^{2} W_{c} \cong \Gamma_{0} \oplus \Gamma_{\mathbf{e}_{2}}
$$

which follows easily using Lemma 3.2. Note that the differential $d$ maps the summand $\Gamma_{\mathbf{e}_{2}}$ to $q_{g-2}^{1} \cdot \wedge_{0}^{2} V^{3}$.

We now proceed to prove the second part of the theorem.

Proof of (i). Let us prove by induction on $k \geq 1$ that there exists a sub-representation $U_{k} \subset V^{2 g+2 k-2}$ with $U_{k} \cong \Gamma_{k} \mathbf{e}_{1}$ such that $d\left(U_{k}\right) \subset U_{k-1}$. $V^{3} \subset V^{2 g+2 k-4} \cdot V^{3}$, where $U_{0}:=\left\langle q_{g-1}^{1}\right\rangle \subset V^{2 g-2}$.

If $k=1$, then

$$
V^{n}=V^{2 g}=\Gamma_{\mathbf{e}_{1}}
$$

with $d: V^{2 g} \longrightarrow q_{g-1}^{1} \cdot V^{3} \subset \bigwedge V$.

Now assume that there exists a sub-representation $U_{k-1} \subset V^{2 g+2 k-4}$ with $U_{k-1} \cong \Gamma_{(k-1) \mathbf{e}_{1}}$ such that $d\left(U_{k-1}\right) \subset U_{k-2} \cdot V^{3} \subset V^{2 g+2 k-6} \cdot V^{3}$. Then 
we have

$$
d\left(U_{k-1} \cdot V^{3}\right) \subset U_{k-2} \cdot V^{3} \cdot V^{3}
$$

On one hand,

$$
\begin{aligned}
& U_{k-1} \cdot V^{3}=U_{k-1} \otimes V^{3} \subset V^{2 g+2 k-4} \otimes V^{3} \\
& U_{k-1} \otimes V^{3} \cong \Gamma_{(k-1) \mathbf{e}_{1}} \otimes \Gamma_{\mathbf{e}_{1}}
\end{aligned}
$$

so there exists $\widetilde{U}_{k} \subset U_{k-1} \cdot V^{3}$ with $\widetilde{U}_{k} \cong \Gamma_{k \mathbf{e}_{1}}$ such that $\widetilde{U}_{k} \subset U_{k-1} \cdot V^{3}$. On the other hand,

$$
U_{k-2} \cdot V^{3} \cdot V^{3} \subset U_{k-2} \otimes \wedge^{2} V^{3} \cong \Gamma_{(k-2) \mathbf{e}_{1}} \otimes \Gamma_{\mathbf{e}_{2}}
$$

does not contain $\Gamma_{k} \mathbf{e}_{1}$. So $d\left(\widetilde{U}_{k}\right)=0$, or in other words, $\widetilde{U}_{k} \subset Z^{2 g+2 k-1}(\bigwedge V)$.

By Remark 4.3, we have $\rho\left(V^{n}\right)=0$ for all $n \geq 5$, where $\rho:(\bigwedge V, d) \longrightarrow$ $\left(H^{*}\left(\mathcal{N}_{X}\right), 0\right)$ is the minimal model. As $U_{k-1} \subset V^{2 g+2 k-4}$, we have $\rho\left(U_{k-1}\right)=$ 0 . Hence $\rho\left(\widetilde{U}_{k}\right)=0$, or in other words, $\widetilde{U}_{k} \subset B^{2 g+2 k-1}(\bigwedge V)$. This is only possible if there exists $U_{k} \subset V^{2 g+2 k-2}$ with $U_{k} \cong \Gamma_{k} \mathbf{e}_{1}$ and $d: U_{k} \stackrel{\simeq}{\longrightarrow} \widetilde{U}_{k} \subset$ $U_{k-1} \cdot V^{3}$. Therefore, the proof of statement (i) is complete by induction.

Proof of (ii). We will show using induction on $k \geq 1$ that there exists a sub-representation

$$
U_{k} \subset V^{2 g+2 k+1}
$$

with $U_{k} \cong \Gamma_{k \mathbf{e}_{1}+\mathbf{e}_{2}}$ such that $d\left(U_{k}\right) \subset U_{k-1} \cdot V^{3} \subset V^{2 g+2 k-1} \cdot V^{3}$, where $U_{0} \subset$ $V^{2 g+1}$ is the sub-representation of $V^{2 g+1}=\Gamma_{0} \oplus \Gamma_{\mathbf{e}_{2}}$ isomorphic to $\Gamma_{\mathbf{e}_{2}}$.

For $k=1$, note that $d: U_{0} \rightarrow q_{g-2}^{1} \cdot \wedge_{0}^{2} V^{3}$. So $d: U_{0} \cdot V^{3} \rightarrow q_{g-2}^{1} \cdot \wedge_{0}^{2} V^{3}$. $V^{3}$, where

$$
\wedge_{0}^{2} V^{3} \cdot V^{3} \cong \wedge_{0}^{3} V^{3} \oplus \gamma \cdot V^{3} \cong \wedge_{0}^{3} W_{c} \oplus W_{c}=\Gamma_{\mathbf{e}_{3}} \oplus \Gamma_{\mathbf{e}_{1}}
$$

As $U_{0} \cong \Gamma_{\mathbf{e}_{2}}$, we conclude that

$$
U_{0} \cdot V^{3} \cong U_{0} \otimes V^{3} \cong \Gamma_{\mathbf{e}_{2}} \otimes \Gamma_{\mathbf{e}_{1}}
$$

contains a sub-representation $\widetilde{U}_{1} \subset U_{0} \cdot V^{3}$ with $\widetilde{U}_{1} \cong \Gamma_{\mathbf{e}_{1}+\mathbf{e}_{2}}$ and $d\left(\widetilde{U}_{1}\right)=0$. Working as in the proof of (i), this yields that there exists $U_{1} \subset V^{2 g+3}$ with $U_{1} \cong \Gamma_{\mathbf{e}_{1}+\mathbf{e}_{2}}$ and $d\left(U_{1}\right)=\widetilde{U}_{1} \subset U_{0} \cdot V^{3}$.

Now assume that $k \geq 2$ and that there exists a sub-representation $U_{k-1}$ $\subset V^{2 g+2 k-1}$ with $U_{k-1} \cong \Gamma_{(k-1) \mathbf{e}_{1}+\mathbf{e}_{2}}$ such that $d\left(U_{k-1}\right) \subset U_{k-2} \cdot V^{3} \subset$ 
$V^{2 g+2 k-3} \cdot V^{3}$. Then we have $d\left(U_{k-1} \cdot V^{3}\right) \subset U_{k-2} \cdot V^{3} \cdot V^{3}$. On one hand,

$$
U_{k-1} \cdot V^{3}=U_{k-1} \otimes V^{3} \cong \Gamma_{(k-1) \mathbf{e}_{1}+\mathbf{e}_{2}} \otimes \Gamma_{\mathbf{e}_{1}}
$$

so there exists $\widetilde{U}_{k} \subset U_{k-1} \cdot V^{3}$ with $\widetilde{U}_{k} \cong \Gamma_{k \mathbf{e}_{1}+\mathbf{e}_{2}}$ such that $\widetilde{U}_{k} \subset U_{k-1} \cdot V^{3}$. On the other hand,

$$
U_{k-2} \cdot V^{3} \cdot V^{3} \subset U_{k-2} \otimes \wedge^{2} V^{3} \cong \Gamma_{(k-2) \mathbf{e}_{1}+\mathbf{e}_{2}} \otimes \Gamma_{\mathbf{e}_{2}}
$$

does not contain $\Gamma_{k \mathbf{e}_{1}+\mathbf{e}_{2}}$. Therefore, $d\left(\widetilde{U}_{k}\right)=0$. Thus there exists $U_{k} \subset$ $V^{2 g+2 k-2}$ with $U_{k} \cong \Gamma_{k} \mathbf{e}_{1}+\mathbf{e}_{2}$ and $d: U_{k} \stackrel{\simeq}{\longrightarrow} \widetilde{U}_{k} \subset U_{k-1} \cdot V^{3}$. This completes the proof of the theorem.

From Theorem 6.3 and 7.1 it follows that for each $g \geq 2$, the rational homotopy group $\pi_{n}\left(\mathcal{N}_{X}\right) \otimes_{\mathbb{Z}} \mathbb{C}$ is non-zero for infinitely many $n$. As noted in the Introduction, this means that the moduli space $\mathcal{N}_{X}$ is rationally hyperbolic for all $g \geq 2$. Therefore,

$$
f(k)=\sum_{i=1}^{\operatorname{dim}_{\mathbb{R}} \mathcal{N}_{X}-1} \operatorname{dim} \pi_{k+i}\left(\mathcal{N}_{X}\right) \otimes_{\mathbb{Z}} \mathbb{Q}
$$

grows faster than any polynomial in $k$.

Remark 7.2. Let $X$ be a smooth irreducible projective complex curve of genus $g \geq 2$. Whereas the minimal model of $\left(H^{*}\left(\mathcal{N}_{X}, \mathbb{C}\right), 0\right)$ has infinitely many $n \in \mathbb{N}$ for which $V^{n} \neq 0$, the minimal model of the algebra $\left(H_{I}^{*}\left(\mathcal{N}_{X}\right.\right.$, $\mathbb{C}), 0$ ) has a very different behavior. Actually, from Equation (3.5) we find that the minimal model of $\left(H_{I}^{*}\left(\mathcal{N}_{X}, \mathbb{C}\right), 0\right)$ is

$$
\left(\bigwedge\left(\alpha, \beta, \gamma, f_{1}, f_{2}, f_{3}\right), d\right), \quad d f_{1}=q_{g}^{1}, d f_{2}=q_{g}^{2}, d f_{3}=q_{g}^{3}
$$

where $\operatorname{deg}(\alpha)=2, \operatorname{deg}(\beta)=4, \operatorname{deg}(\gamma)=6, \operatorname{deg}\left(f_{1}\right)=2 g-1, \operatorname{deg}\left(f_{2}\right)=2 g+$ 1 and $\operatorname{deg}\left(f_{3}\right)=2 g+3$.

\section{Acknowledgments}

The authors are grateful to Aniceto Murillo for useful comments. The firstnamed author wishes to thank the Harish-Chandra Research Institute for its hospitality. The second-named author was partially supported through grant MCyT (Spain) MTM2004-07090-C03-01. 


\section{References}

[1] M.F. Atiyah and R. Bott, The Yang-Mills equations over Riemann surfaces, Philos. Trans. Roy. Soc. London Ser. A 308 (1983), 523-615.

[2] I. Biswas, Determinant bundle over the universal moduli space of vector bundles over the Teichmüller space, Ann. Inst. Fourier 47 (1997), 885914.

[3] A. Borel, Density properties for certain subgroups of semi-simple groups without compact components, Ann. of Math. 72 (1960), 179-188.

[4] P. Deligne, Equations différentielles à points singuliers réguliers, Lecture Notes in Mathematics 163, Springer-Verlag, Berlin, NY, 1970.

[5] P. Deligne, P. Griffiths, J. Morgan and D. Sullivan, Real homotopy theory of Kähler manifolds, Invent. Math. 29 (1975), 245-274.

[6] Y. Félix, La dichotomie elliptique-hyperbolique en homotopie rationnelle, Astérisque 179 Société Mathématique de France, Paris, 1989.

[7] W. Fulton and J. Harris, Representation theory. A first course, Graduate Texts in Mathematics 129, Springer-Verlag, Berlin, NY, 1991.

[8] P. Griffiths and J.W. Morgan, Rational homotopy theory and differential forms, Progress in Mathematics 16, Birkhäuser, Boston, MA, 1981.

[9] A.D. King and P.E. Newstead, On the cohomology ring of the moduli space of rank 2 vector bundles on a curve, Topology 37 (1998), 407-418.

[10] J.E. Humphreys, Introduction to Lie algebras and representation theory, Graduate Texts in Mathematics 9, Springer-Verlag, Berlin, NY, 1978.

[11] V. Muñoz, Quantum cohomology of the moduli space of stable bundles over a Riemann surface, Duke Math. J. 98 (1999), 525-540.

[12] D. Mumford and P.E. Newstead, Periods of a moduli space of bundles on curves, Amer. J. Math. 90 (1968), 1200-1208.

[13] M.S. Narasimhan and S. Ramanan, Moduli of vector bundles on a compact Riemann surface, Ann. of Math. 89 (1969), 14-51.

[14] M.S. Narasimhan and C.S. Seshadri, Stable and unitary vector bundles on a compact Riemann surface, Ann. Math. 82 (1965), 540-567. 
[15] V. Navarro Aznar, Sur la connexion de Gauss-Manin en homotopie rationnelle, Ann. Sci. École Norm. Sup. 26 (1993), 99-148.

[16] P.E. Newstead, Topological properties of some spaces of stable bundles, Topology 6 (1967), 241-262.

[17] P.E. Newstead, Stable bundles of rank 2 and odd degree over a curve of genus 2, Topology 7 (1968), 205-215.

[18] P.E. Newstead, Characteristic classes of stable bundles of rank 2 over an algebraic curve, Trans. Am. Math. Soc. 169 (1972), 337-345.

[19] P.E. Newstead, Introduction to moduli problems and orbit spaces, T.I.F.R. Lectures on Mathematics and Physics, 51, Narosa Publishing House, New Delhi, 1978.

[20] B. Siebert and G. Tian, Recursive relations for the cohomology ring of moduli spaces of stable bundles, Turkish J. Math. 19 (1995), 131-144.

[21] M. Thaddeus, Conformal field theory and the cohomology of the moduli space of stable bundles, J. Diff. Geom. 35 (1992), 131-150.

SCHOOL OF MATHEMATiCS

Tata Institute of Fundamental Research

HOMI BHABHA ROAD

BOMBAY 400005

INDIA

E-mail address: indranil@math.tifr.res.in

Departamento de Matemáticas

Consejo Superior de Investigaciones Cientificas, Serrano 113 Bis

28006 MADRID

SPAIN

Facultad de Matemáticas

Universidad Complutense de Madrid

Plaza de Ciencias 3

28040 MADRID

SPAIN

E-mail address: vicente.munoz@imaff.cfmac.csic.es

Received September 21, 2007 